\title{
Adicciones a internet y funciones ejecutivas en estudiantes universitarios: una revisión sistemática
}

\section{Elena Bernabéu ${ }^{1}$, Carlos A. Marchena ${ }^{1}$, Adriana González-Pizzio $^{1}$, Genny Lubrini ${ }^{2}$}

${ }^{1}$ Universidad Francisco de Vitoria, Pozuelo, Madrid

${ }^{2}$ Universidad Complutense de Madrid

Correspondencia: Elena Bernabéu Brotóns. Facultad de Psicología y Educación. Universidad Francisco de Vitoria, carretera Pozuelo Majadahonda km 1.8, 28223 Madrid. España. E-mail: e.bernabeu.prof@ufv.es

(C) Universidad de Almería and Ilustre Colegio Oficial de la Psicología de Andalucía Oriental (Spain)) 


\section{Resumen}

La adicción a internet es un problema de salud pública cada vez mayor, y los estudiantes universitarios reúnen una serie de características que les convierte en población de riesgo. Numerosos estudios sugieren una relación entre adicciones y alteración en las funciones ejecutivas. Se presenta una revisión bibliográfica que tiene por objetivo identificar variables neuropsicológicas de riesgo para el desarrollo de la adicción a las diferentes aplicaciones de la web en la etapa universitaria. Se realizó una búsqueda sistemática de estudios empíricos publicados entre 2000 y 2019 sobre la relación entre adicción a internet y función ejecutiva en esta población a través de bases de datos online (Medline, PsycInfo, PubMED, ScienceDirect, Scopus y Web of Science). Tras eliminar registros duplicados y aplicar criterios de elegibilidad, se seleccionaron 30 artículos. Los resultados sugieren la implicación de las funciones ejecutivas (control inhibitorio, toma de decisiones y fluidez verbal), aunque no permiten establecer un perfil de riesgo claro para el desarrollo de adicciones a internet. Parece necesario profundizar en la naturaleza de esta relación, diferenciando entre las diferentes aplicaciones y controlando variables como el género, la naturaleza de la tarea y el tipo de estímulo, con el fin de elaborar estrategias preventivas eficaces.

Palabras Clave: adicción a internet, funciones ejecutivas, toma de decisiones, fluidez verbal, estudiantes universitarios. 


\begin{abstract}
Internet addiction is becoming a growing public health problem, and it particularly can affect college students, who gather features that make them population at risk. Numerous studies have suggested the relationship between addictive behaviours and an alteration of executive functions. It is provided a comprehensive overview of the scientific literature on internet addiction and executive functions in college students in last 19 years. The aim of the study is identifying neuropsychological risk variables for the development of addiction to the different uses or applications of the web in the university stage. It was conducted a systematic search of empirical studies published between 2000 and 2019 that had addressed the relationship between internet addiction and executive function in this population through online databases (Medline, PsycInfo, PubMED, ScienceDirect, SCOPUS and Web of Science). After deleting duplicate records and applying eligibility criteria it was selected 30 publications. The results suggest the involvement of executive functions (specifically inhibitory control, decision making and verbal fluency), although they do not allow establishing a clear risk profile. It seems necessary to carry out further investigation about the nature of this relationship, differentiating between the diverse applications of the internet, controlling variables such as gender, the nature of the task and the type of stimuli, in order to develop effective programs in prevention and treatment.
\end{abstract}

Keywords: internet addiction, executive functions, verbal fluency, college students. 


\section{Introducción}

Se ha definido la adicción o uso problemático de internet como un trastorno mental, comportamental y social relacionado con el uso repetido y excesivo de internet (Tao et al., 2008), caracterizado por una excesiva preocupación, conducta impulsiva o falta de control con respecto al acceso a la red (Shaw y Black, 2015) y que acarrea consecuencias negativas en el desempeño de la vida diaria (Spada, 2014).

A pesar del creciente interés científico por este trastorno, la formulación conceptual y criterios diagnósticos de la adicción a internet siguen siendo objeto de controversia (Kuss, Griffiths, Karila y Billieux, 2014). El trastorno por adicción a internet no ha sido incluido hasta la fecha en el Manual Diagnóstico y Estadístico de los Trastornos Mentales (DSM-5; APA, 2013), aunque la incorporación del apartado "trastornos adictivos no relacionados con sustancias" (DSM-5 appendix; section III, emerging disorders) constituye un acercamiento al reconocimiento oficial del problema. Una propuesta reciente establece como criterios diagnósticos de la adicción a internet: preocupación por acceder a internet, aislamiento social, craving, pérdida de control sobre el acceso a internet, uso excesivo de la web, pérdida de interés general y escape de la realidad en un estado disfórico (Kuss y Lopez-Fernandez, 2016). Del mismo modo, tampoco existe un acuerdo en la referente a la terminología utilizada para describir este problema. Aunque la mayor parte de los autores hablan de adicción a internet (Chou et al., 2005; Hansen, 2002; Widyanto y Griffiths, 2006; Young, 1998, 2004; Young, Yue y Ying, 2011), también se ha utilizado uso compulsivo de internet (Meerkerk, Van Den Eijnden, Franken y Garretsen, 2010; Meerkerk, Van Den Eijnden, y Garretsen, 2006; Meerkerk, Van Den Eijnden, Vermulst y Garretsen, 2009), uso problemático de internet (Caplan, 2002), y uso patológico de internet (Davis, 2001). En adelante se utilizará el término adicción a internet por ser el más utilizado en la literatura y por los evidentes paralelismos con la adicción a sustancias (Meerkerk, Van Den Eijnden, Vermulst y Garretsen, 2009). Existen ciertos mecanismos y alteraciones similares entre este tipo de dependencia y otro tipo de conductas adictivas, como las relacionadas con los juegos de azar, el sexo, las compras, la comida y el uso de internet (Andreassen, Pallesen, Griffiths, Torsheim y Sinha, 2018; Jorgenson, Hsiao y Yen, 2016; Luengo-López, 2004). En concreto, la adicción a internet se acompaña de modificación del estado de ánimo, retraimiento social y conflicto interno (Griffiths, 2005), estados de estrés, 
sintomatología depresiva y uso de estrategias de afrontamiento negativas (Hasan y Jaber, 2019; Park, Hong, Park, Ha, y Yoo, 2013), así como falta de sueño, bajo rendimiento académico, problemas de atención y tendencia al establecimiento de objetivos a corto plazo (Aboujaoude, 2010; Wu, Cheung, Ku, y Hung, 2013).

En relación con los procesos cognitivos, la adicción a sustancias se ha relacionado con una alteración de las funciones ejecutivas. El término funciones ejecutivas engloba una serie de procesos de alto nivel (tales como control inhibitorio, memoria de trabajo y flexibilidad cognitiva) necesarios para llevar a cabo actividades dirigidas a un objetivo, y que permiten emitir respuestas a situaciones novedosas o complejas y cuyo sustrato neuroanatómico se sitúa en la cortez prefrontal (Bausela, 2014). También incluye la toma de decisiones (Tranel y Damasio, 2000), que requiere la integración de numerosos procesos cognitivos y emocionales (Martínez-Selva,. Sánchez-Navarro, Bechara y Román). Muy relacionada con el funcionamiento ejecutivo está la denominada velocidad de procesamiento, que más que un proceso cognitivo es una propiedad del sistema de procesamiento, y que se refiere a la cantidad de información que puede procesarse por unidad de tiempo (Ríos-Lago, Periañez y MuñozCéspedes, 2004). La alteración ejecutiva asociada con el consumo de sustancias se caracteriza concretamente por una toma de decisiones sesgada hacia las recompensas inmediatas, así como por un control inhibitorio reducido (Hekmat, Mehrjerdi, Moradi, Ekhtiari y Bakhshi, 2011). Estas dificultades cognitivas están presentes también en las adicciones comportamentales: se ha encontrado alteraciones en la toma de decisiones y reducción en el control atencional en la adicción a la comida (Steward et al., 2018), falta de flexibilidad cognitiva y comportamiento impulsivo en jugadores patológicos (Marazziti et al., 2008) y dificultades de control inhibitorio en el trastorno hipersexual (Seok y Sohn, 2018). Del mismo modo, se ha descrito la existencia de problemas de control ejecutivo en las personas con adicción a internet (Billieux y Van der Linden, 2012).

La adicción a internet incluye la realización compulsiva de compras a través de la web, el acceso a contenidos pornográficos, el juego a través de plataformas o redes sociales y las apuestas online (Ioannidis et al., 2016; Király, Griffiths y Demetrovics, 2015). La adicción a internet podría por tanto entenderse como diferentes adicciones de contenido específico. En este sentido Davis (2001) distingue entre adicción generalizada 
a internet y adicción a los contenidos de aplicaciones específicas. En este último caso, la conducta problemática debería darse también fuera de internet, aunque la facilidad de acceso que proporciona la web podría agravar el problema. Aun asumiendo que la adicción a cada aplicación pudiera tener sus propias peculiaridades, en todos los casos se reconocen los criterios de adicción a internet ya descritos (Kuss y Lopez-Fernandez, 2016).

La adicción a internet se está convirtiendo en un grave problema de salud pública, y es cada vez más frecuente entre los más jóvenes (Kuss et al., 2014), con una mayor prevalencia entre los universitarios, entre los que se encuentra la mayor proporción de personas con un uso problemático y abusivo de internet (Hsieh, Hsiao, Yang, Lee y Yen, 2019; Krishnamurthy y Chetlapalli, 2018). Los estudiantes universitarios reúnen una serie de características que les convierte en población de riesgo: internet es una de las principales herramientas en las actividades académicas, lo que requiere y justifica una gran cantidad de tiempo online; además los horarios de estudio y trabajo de los estudiantes universitarios son muy flexibles, existiendo poca supervisión de los adultos sobre sus actividades en la web. Todo esto favorece el uso sin control de internet, participación en juegos, realización de apuestas, compras online o consumo de pornografía (Kuss, Griffiths y Binder, 2013). Estudios de prevalencia realizados con esta población sugieren que un número importante de los estudiantes universitarios muestran adicción a internet en alguna de sus modalidades, con porcentajes que oscilan entre un 3,2\% (Kuss et al., 2013) y un 11\% (Shao et al., 2018).

Los estudiantes universitarios se encuentran además en un momento especialmente importante del neurodesarrollo, que corresponde al final de la maduración del lóbulo prefrontal (García-Moreno, Expósito, Sanhueza y Angulo, 2008), que tiene lugar entre el final de la adolescencia y el inicio de la edad adulta (Blakemore y Choudhury, 2006; Casey, Giedd y Thomas, 2000), etapa caracterizada por el establecimiento de circuitos neuronales cada vez más complejos que constituirán el correlato neurofisiológico de las funciones ejecutivas (Gogtay et al., 2004). La aparición de conductas adictivas en esta etapa de la vida podría interferir y/o alterar el adecuado el desarrollo cerebral. La adolescencia y primera juventud es, además, un momento de mayor vulnerabilidad para el desarrollo de adicciones, debido a un control cognitivo aún 
no plenamente desarrollado y por tanto poco efectivo (Casey, Tottenham, Liston y Durston, 2005) y a dificultades para establecer límites (Liu y Potenza, 2007).

Parece necesario clarificar y profundizar en la relación existente entre la adicción a internet y la disfunción ejecutiva en estudiantes universitarios con el fin de responder a la siguiente pregunta ¿existen disfunciones ejecutivas características de la adicción a internet en la etapa universitaria? La identificación de un perfil cognitivo de riesgo que implique una mayor vulnerabilidad permitirá implementar estrategias de prevención e intervención más efectivas. Con este objetivo se llevó a cabo una revisión bibliográfica sistemática de estudios empíricos realizados en la última década.

\section{Método}

La presente revisión bibliográfica se ha realizado siguiendo los postulados de la declaración PRISMA. Se consultaron bases de datos online (Medline, PsycInfo, PubMED, ScienceDirect, SCOPUS y Web of Science) con el objetivo de realizar una revisión sistemática de publicaciones empíricas que examinasen la relación entre la adicción a internet o a aplicaciones específicas de internet (videojuegos, compras, apuestas y/o pornografía online) y el funcionamiento ejecutivo. La búsqueda se limitó al período 2000-2019, entendiendo que ha sido en esta última década cuando el acceso a internet está plenamente extendido en la población, debido en parte al uso generalizado del teléfono inteligente o smartphone para tal fin. Los términos utilizados fueron: "adicción a internet", "adicción a los videojuegos online", "adicción a las compras online", "adicción a la pornografía online", "adicción a las apuestas online" y "funciones ejecutivas", "velocidad de procesamiento", "fluidez verbal", “inhibición", "memoria de trabajo", "flexibilidad cognitiva", "toma de decisiones", tanto en castellano como en inglés, combinándose entre ellos mediante los operadores booleanos AND y OR. Aunque algunos autores argumentan contra la inclusión de la fluidez verbal dentro de las funciones ejecutivas, considerando que esta tarea refleja el procesamiento del lenguaje (Whiteside et al., 2016), para otros el factor críticamente implicado en esta tarea es un componente ejecutivo (Aita et al., 2016; Hirshorn y Thompson-Shill, 2006), y por eso se ha incluido el término "fluidez verbal" en esta revisión. 
Criterios de elegibilidad: se seleccionaron estudios empíricos escritos en inglés o en español que explorasen la relación entre la adicción a internet y las funciones ejecutivas realizados con estudiantes universitarios desde la neuropsicología, neurociencia o psicología. Los criterios de exclusión fueron: artículos de revisión, capítulos de libro, artículos publicados en otros idiomas, estudios sin relación con la temática de interés, estudios cuyos participantes no fuesen estudiantes universitarios o tuviesen otra patología diagnosticada, aquellos en los que no se especificase una medida de la adicción a internet y/o prueba neuropsicológica para valorar las funciones ejecutivas, estudios que se centraran exclusivamente en técnicas de neuroimagen, biomarcadores y estudios genéticos. Finalmente se encontraron 11069 registros en bases de datos y 12 registros de referencias secundarias, lo que supone un total de 11081, de los cuales 290 se correspondieron con aquellos devueltos por Medline, 1208 por PsycInfo, 415 por PubMED, 7990 por ScienceDirect, 237 por SCOPUS y 929 por Web of Science (WoS). De este total se eliminaron 459 por estar duplicados y se rechazaron 10592 por no cumplir los criterios de elegibilidad, tal como se muestra en la figura 1.

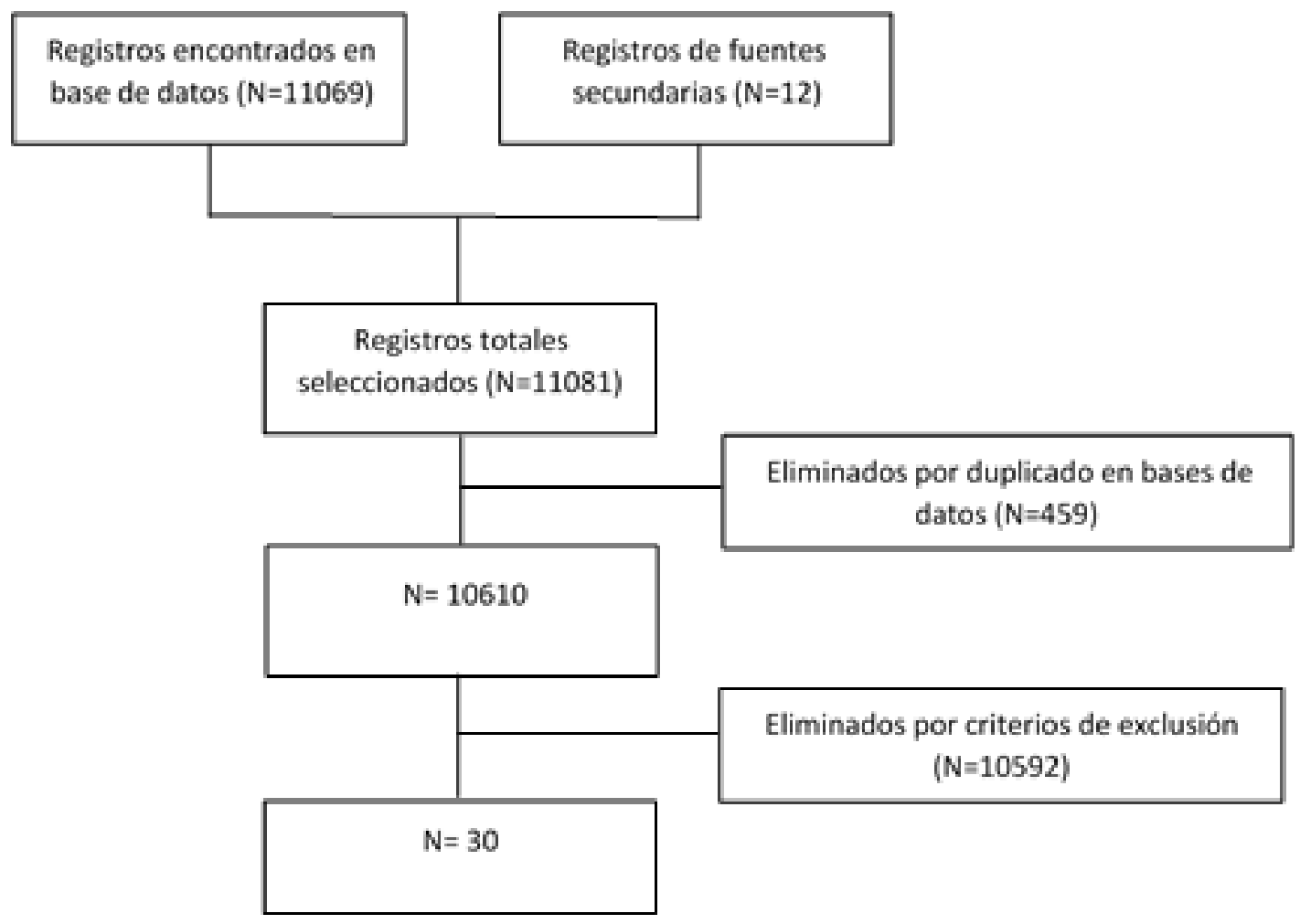

Figura 1. Diagrama de flujo de la revisión. 


\section{Resultados}

En la tabla 1 se exponen los resultados de los estudios revisados, atendiendo a las características de la muestra, tipo de adicción a Internet (aplicación de la que se hace un uso problemático), los instrumentos utilizados para valorar la adicción y las funciones ejecutivas, el tipo de análisis aplicado y, por último, los principales resultados y conclusiones relevantes.

Tabla 1.Resultados encontrados en la revisión sistemática

\begin{tabular}{|c|c|c|c|c|c|c|c|c|c|}
\hline $\begin{array}{l}\text { Referencia } \\
\text { del estudio }\end{array}$ & Muestra & $\begin{array}{l}\text { Subtipo } \\
\text { de AI }\end{array}$ & $\begin{array}{l}\text { Instrumento } \\
\text { de valoración } \\
\text { AI }\end{array}$ & $\begin{array}{l}\text { FFEE } \\
\text { evaluada }\end{array}$ & $\begin{array}{l}\text { Instrumento } \\
\text { de valoración } \\
\text { FFEE }\end{array}$ & $\begin{array}{l}\text { Otras } \\
\text { pruebas } \\
\text { aplicadas }\end{array}$ & $\begin{array}{l}\text { Análisis } \\
\text { realizados }\end{array}$ & Resultados & Conclusión \\
\hline $\begin{array}{l}\text { Sun et al., } \\
\text { (2009) }\end{array}$ & $\begin{array}{l}113 \text { estudiantes } \\
\text { universitarios } \\
-52 \text { adictos } \\
-61 \text { controles }\end{array}$ & General & IAT & $\begin{array}{l}\text { Toma de } \\
\text { decisiones. } \\
\text { Control } \\
\text { inhibitorio }\end{array}$ & $\begin{array}{l}\text { Modified } \\
\text { Gambling Task } \\
\text { Go/no-Go task }\end{array}$ & & $\begin{array}{l}\text { ANOVA } \\
\text { Post-hoc }\end{array}$ & $\begin{array}{l}\text { Grupo AI obtiene } \\
\text { puntuación menor total ( } p \\
=.003 \text { ) en Gambling Task, } \\
\text { aunque obtiene mayores } \\
\text { puntuaciones en los } \\
\text { últimos bloques }(p<.001) \\
\text { No diferencias entre } \\
\text { grupos en la condición Go, } \\
\text { pero mayor precisión de } \\
\text { respuesta de los adictos en } \\
\text { la condición no-Go ( } p \\
=.018) .\end{array}$ & $\begin{array}{l}\text { Las personas con AI: } \\
\text { - Existe un déficit en la } \\
\text { toma de decisiones, } \\
\text { caracterizado por un } \\
\text { retraso, más que por una } \\
\text { incapacidad de aprender } \\
\text { de las contingencias } \\
\text { (menor velocidad de } \\
\text { cambio de estrategia). } \\
\text { - No hay déficit } \\
\text { inhibitorio en la tarea } \\
\text { Go/no Go, a pesar de la } \\
\text { falta de control sobre el } \\
\text { acceso a internet. }\end{array}$ \\
\hline $\begin{array}{l}\text { Dong, Zhou } \\
\text { y Zhao } \\
\text { (2010) }\end{array}$ & $\begin{array}{l}24 \text { estudiantes } \\
\text { universitarios } \\
\text { hombres } \\
\text { - } 12 \text { AI } \\
\text { - } 12 \text { Controles }\end{array}$ & General & IAT & $\begin{array}{l}\text { Control } \\
\text { inhibitorio }\end{array}$ & Go/no-Go task & & t-Student & $\begin{array}{l}\text { No hay diferencias entre } \\
\text { grupos en precisión ni } \\
\text { velocidad de respuesta en } \\
\text { ninguna de las dos } \\
\text { condiciones (Go/ no-Go). }\end{array}$ & $\begin{array}{l}\text { Las personas con AI no } \\
\text { muestran alteración en el } \\
\text { control inhibitorio. }\end{array}$ \\
\hline
\end{tabular}


Tabla 2 (continua).Resultados encontrados en la revisión sistemática

\begin{tabular}{|c|c|c|c|c|c|c|c|c|c|}
\hline $\begin{array}{l}\text { Referencia } \\
\text { del estudio }\end{array}$ & Muestra & $\begin{array}{l}\text { Subtipo } \\
\text { de AI }\end{array}$ & $\begin{array}{l}\text { Instrumento } \\
\text { de valoración } \\
\text { AI }\end{array}$ & $\begin{array}{l}\text { FFEE } \\
\text { evaluada }\end{array}$ & $\begin{array}{l}\text { Instrumento } \\
\text { de valoración } \\
\text { FFEE }\end{array}$ & $\begin{array}{l}\text { Otras } \\
\text { pruebas } \\
\text { aplicadas }\end{array}$ & $\begin{array}{l}\text { Análisis } \\
\text { realizados }\end{array}$ & Resultados & Conclusión \\
\hline $\begin{array}{l}\text { Ko et al., } \\
\text { (2010) }\end{array}$ & $\begin{array}{l}188 \text { estudiantes } \\
\text { universitarios } \\
-74 \mathrm{AI} \\
-114 \text { Controles }\end{array}$ & General & DCIA-C & $\begin{array}{l}\text { Toma de } \\
\text { decisones }\end{array}$ & $\begin{array}{l}\text { Iowa Gambling } \\
\text { Task } \\
\text { Ballon Analoge } \\
\text { Risk Task }\end{array}$ & TPQ & $\begin{array}{l}\text { t-Student } \\
\text { MANOVA }\end{array}$ & $\begin{array}{l}\text { IGT: El grupo AI escoge } \\
\text { cartas del montón } \\
\text { desventajoso en los } \\
\text { primeros } 40 \text { ensayos } \\
(p=.035) \text { y del montón } \\
\text { ventajoso en los últimos } \\
40(\mathrm{p}=.001) \text {. } \\
\text { No hubo diferencias entre } \\
\text { grupos en la tarea BART }\end{array}$ & $\begin{array}{l}\text { Las personas con AI: } \\
\text { - Aprendizaje emocional } \\
\text { implícito mejor que } \\
\text { niños no adictos } \\
\text { (aprendizaje en la tarea } \\
\text { IGT). } \\
\text { - En general no toman } \\
\text { decisiones más } \\
\text { arriesgadas. } \\
\text { - Les caracteriza la } \\
\text { búsqueda de la novedad. }\end{array}$ \\
\hline $\begin{array}{l}\text { Saville et al., } \\
\text { (2010) }\end{array}$ & $\begin{array}{l}28 \text { estudiantes } \\
\text { universitarios } \\
-14 \text { AI } \\
-14 \text { Controles }\end{array}$ & General & IAT & $\begin{array}{l}\text { Toma de } \\
\text { decisiones. } \\
\text { Control } \\
\text { inhibitorio }\end{array}$ & $\begin{array}{l}\text { Delay } \\
\text { Discounting } \\
\text { Task }\end{array}$ & & t-Student & $\begin{array}{l}\text { El grupo con AI busca la } \\
\text { recompensa inmediata } \\
\text { aunque sea de menor } \\
\text { cuantía a diferencia del } \\
\text { grupo control }(p=.02) \text {. }\end{array}$ & $\begin{array}{l}\text { Las personas con AI } \\
\text { presentan mayor } \\
\text { impulsividad. }\end{array}$ \\
\hline $\begin{array}{l}\text { Dong et al., } \\
\text { (2011) }\end{array}$ & $\begin{array}{l}34 \text { estudiantes } \\
\text { universitarios } \\
\text { hombres } \\
-17 \text { AI } \\
-17 \text { Controles }\end{array}$ & General & IAT & $\begin{array}{l}\text { Control } \\
\text { inhibitorio }\end{array}$ & Stroop Task & & ANOVA & $\begin{array}{l}\text { El grupo AI es más lento } \\
\text { en la condición } \\
\text { incongruente }(p<.01) * \text {, y } \\
\text { comete más errores } \\
(\mathrm{p}=0.01) \text {. }\end{array}$ & $\begin{array}{l}\text { Las personas con AI } \\
\text { presentan menor capacidad } \\
\text { de control inhibitorio. }\end{array}$ \\
\hline $\begin{array}{l}\text { Pawllikoswki } \\
\text { et al., (2011) }\end{array}$ & $\begin{array}{l}38 \text { estudiantes } \\
\text { universitarios } \\
-\quad 19 \text { AI (juego) } \\
-\quad 19 \text { Controles }\end{array}$ & $\begin{array}{l}\text { Juego on } \\
\text { line }\end{array}$ & $\begin{array}{l}\text { IAT } \\
\text { SICWoW }\end{array}$ & $\begin{array}{l}\text { Toma de } \\
\text { decisiones }\end{array}$ & $\begin{array}{l}\text { Game of Dice } \\
\text { Task (GDT) }\end{array}$ & SCL90-R & $\begin{array}{l}\text { t-Student } \\
\text { Correlación } \\
\text { ANOVA } \\
\text { medidas } \\
\text { repetidas }\end{array}$ & $\begin{array}{l}\text { Los jugadores tienen un peor } \\
\text { desempeño en la tarea. } \\
(\mathrm{p}=.002) \text {, toman más } \\
\text { decisiones arriesgadas } \\
(\mathrm{p}=.005) \text { y menos decisiones } \\
\text { seguras que el grupo control } \\
(\mathrm{p}=0.01) \text {. } \\
\text { La puntuación total del GDT } \\
\text { correlaciona de forma inversa } \\
\text { con la puntuación obtenida en } \\
\text { el IAT ( } \mathrm{p}=.026) \text {. }\end{array}$ & $\begin{array}{l}\text { Las personas con adicción } \\
\text { al juego por internet } \\
\text { presentan una alteración en } \\
\text { la toma de decisiones. } \\
\text { Hay una relación directa } \\
\text { entre la severidad de la } \\
\text { adicción y el déficit en la } \\
\text { toma de decisiones. }\end{array}$ \\
\hline
\end{tabular}


Tabla 3 (continua).Resultados encontrados en la revisión sistemática

\begin{tabular}{|c|c|c|c|c|c|c|c|c|c|}
\hline $\begin{array}{l}\text { Referencia } \\
\text { del estudio }\end{array}$ & Muestra & $\begin{array}{l}\text { Subtipo } \\
\text { de AI }\end{array}$ & $\begin{array}{l}\text { Instrumento } \\
\text { de valoración } \\
\text { AI }\end{array}$ & $\begin{array}{l}\text { FFEE } \\
\text { evaluada }\end{array}$ & $\begin{array}{l}\text { Instrumento } \\
\text { de valoración } \\
\text { FFEE }\end{array}$ & $\begin{array}{l}\text { Otras } \\
\text { pruebas } \\
\text { aplicadas }\end{array}$ & $\begin{array}{l}\text { Análisis } \\
\text { realizados }\end{array}$ & Resultados & Conclusión \\
\hline $\begin{array}{l}\text { Littel et al., } \\
\text { (2012) }\end{array}$ & $\begin{array}{l}52 \text { estudiantes } \\
\text { universitarios } \\
\text { - } 25 \text { AI (juego) } \\
\text { - } 27 \text { Control }\end{array}$ & $\begin{array}{l}\text { Juego on } \\
\text { line }\end{array}$ & VAT & $\begin{array}{l}\text { Control } \\
\text { inhibitorio }\end{array}$ & Go/no-Go task & EIS & $\begin{array}{l}\text { ANOVA } \\
\text { Post-Hoc } \\
\text { Correlación }\end{array}$ & $\begin{array}{l}\text { Condición no-Go }(\mathrm{p}<.01) \text { : } \\
\text { más errores del grupo de } \\
\text { adictos al juego online } \\
\text { Condición no Go: más } \\
\text { falsas alarmas }(\mathrm{p}<.01) \text { y } \\
\text { menor TR }(\mathrm{p}<.05) . \\
\text { Los falsos positivos } \\
\text { correlacionan con las } \\
\text { horas de juego semanal } \\
(\mathrm{p}<.05)^{*} .\end{array}$ & $\begin{array}{l}\text { Las personas con adicción } \\
\text { al juego on line presentan } \\
\text { peor control inhibitorio. } \\
\text { Cuanto más grave es la } \\
\text { adicción peor es el control } \\
\text { inhibitorio }\end{array}$ \\
\hline $\begin{array}{l}\text { Bailey et al., } \\
\text { (2013) }\end{array}$ & $\begin{array}{l}149 \text { estudiantes } \\
\text { universitarios } \\
-70 \text { mujeres } \\
-79 \text { hombres }\end{array}$ & $\begin{array}{l}\text { Juego on } \\
\text { line }\end{array}$ & $\begin{array}{l}\text { MUC } \\
\text { PVP }\end{array}$ & $\begin{array}{l}\text { Toma de } \\
\text { decisiones }\end{array}$ & $\begin{array}{l}\text { Iowa Gambling } \\
\text { Task } \\
\text { Temporal } \\
\text { Discounting } \\
\text { Task }\end{array}$ & $\begin{array}{l}\text { BIS-11 } \\
\text { RAS }\end{array}$ & $\begin{array}{l}\text { Correlación } \\
\text { canónica }\end{array}$ & $\begin{array}{l}\text { Existe correlación inversa } \\
\text { entre la interacción de } \\
\text { horas de juego y gravedad } \\
\text { del trastorno y el } \\
\text { desempeño en la IGT } \\
(p<.05) \text { tanto en el TR } \\
(p<.05) \text { como en la } \\
\text { selección de decisiones no } \\
\text { arriesgadas ( } p<.05)^{*} \text {. } \\
\text { El no de horas correlaciona } \\
\text { con la impulsividad en la } \\
\text { adicción a juegos de } \\
\text { disparos (FPS games) pero } \\
\text { no en los juegos de } \\
\text { estrategia, donde la } \\
\text { correlación es inversa. }\end{array}$ & $\begin{array}{l}\text { La mayor gravedad y } \\
\text { mayor tiempo en juegos on } \\
\text { line: } \\
\text { - Menor aprendizaje en la } \\
\text { tarea de toma de } \\
\text { decisiones. } \\
\text { - Mayor número de } \\
\text { decisiones arriesgadas. } \\
\text { La relación entre adicción } \\
\text { al juego, impulsividad y } \\
\text { toma de decisiones } \\
\text { arriesgadas depende del } \\
\text { tipo de juego (disparos } \\
\text { lestrategia): los jugadores } \\
\text { de estrategia no muestran } \\
\text { impulsividad ni decisiones } \\
\text { arriesgadas. }\end{array}$ \\
\hline $\begin{array}{l}\text { Dong et al., } \\
\text { (2014) }\end{array}$ & $\begin{array}{l}30 \text { estudiantes } \\
\text { universitarios } \\
\text { hombres } \\
\text { - } 15 \text { AI } \\
-15 \text { Controles }\end{array}$ & General & IAT & $\begin{array}{l}\text { Control } \\
\text { inhibitorio }\end{array}$ & Stroop Task & MINI & $\begin{array}{l}\text { ANOVA } \\
\text { Medidas } \\
\text { repetidas }\end{array}$ & $\begin{array}{l}\text { El grupo AI tiene peor } \\
\text { desempeño en la tarea, } \\
\text { aunque las diferencias no } \\
\text { son significativas. }\end{array}$ & $\begin{array}{l}\text { La AI no se relaciona con } \\
\text { un peor control inhibitorio. }\end{array}$ \\
\hline
\end{tabular}




\section{Tabla 4 (continua).Resultados encontrados en la revisión sistemática}

\begin{tabular}{|c|c|c|c|c|c|c|c|c|c|}
\hline $\begin{array}{l}\text { Referencia } \\
\text { del estudio }\end{array}$ & Muestra & $\begin{array}{l}\text { Subtipo } \\
\text { de AI }\end{array}$ & $\begin{array}{l}\text { Instrumento } \\
\text { de valoración } \\
\text { AI }\end{array}$ & $\begin{array}{l}\text { FFEE } \\
\text { evaluada }\end{array}$ & $\begin{array}{l}\text { Instrumento } \\
\text { de valoración } \\
\text { FFEE }\end{array}$ & $\begin{array}{l}\text { Otras } \\
\text { pruebas } \\
\text { aplicadas }\end{array}$ & $\begin{array}{l}\text { Análisis } \\
\text { realizados }\end{array}$ & Resultados & Conclusión \\
\hline $\begin{array}{l}\text { Xing, et al. } \\
\text { (2014) }\end{array}$ & $\begin{array}{l}34 \text { estudiantes } \\
\text { universitarios } \\
-17 \text { AI (juego) } \\
-17 \text { Controles }\end{array}$ & $\begin{array}{l}\text { Juego on } \\
\text { line }\end{array}$ & IAT & $\begin{array}{l}\text { Control } \\
\text { inhibitorio }\end{array}$ & Stroop Task & & t-Student & $\begin{array}{l}\text { El grupo de adictos al } \\
\text { juego comete más errores } \\
\text { durante la condición } \\
\text { incongruente }(p<.05) \text { y su } \\
\text { TR total es mayor } \\
(p<.05)^{*} \text {. }\end{array}$ & $\begin{array}{l}\text { Las personas con adicción } \\
\text { a los juegos on line } \\
\text { muestran menor control } \\
\text { cognitivo. }\end{array}$ \\
\hline $\begin{array}{l}\text { Yao et al., } \\
\text { (2014) }\end{array}$ & $\begin{array}{l}52 \text { estudiantes } \\
\text { universitarios } \\
-26 \text { AI (juego) } \\
\text {-26 Controles }\end{array}$ & $\begin{array}{l}\text { Juego on } \\
\text { line }\end{array}$ & CIAS & $\begin{array}{l}\text { Toma de } \\
\text { decisiones }\end{array}$ & $\begin{array}{l}\text { Game of Dice } \\
\text { Task original y } \\
\text { modificado }\end{array}$ & & $\begin{array}{l}\text { t-Student } \\
\text { ANOVA } \\
\text { Correlación }\end{array}$ & $\begin{array}{l}\text { Los adictos al juego } \\
\text { obtienen puntuaciones } \\
\text { netas y totales más bajas } \\
(p<.01) \text { y acumulan menos } \\
\text { dinero }(p<.001) \text {. } \\
\text { La puntuación del CIAS } \\
\text { se relaciona de forma } \\
\text { inversa con las } \\
\text { puntuaciones netas } \\
(p<.001) \text { y totales de la } \\
\text { tarea GDT }(p<.001)^{*} .\end{array}$ & $\begin{array}{l}\text { Los adictos al juego on line } \\
\text { - Rinden peor en la } \\
\text { prueba de toma de } \\
\text { decisiones. } \\
\text { - Toma de decisiones } \\
\text { segadas hacia los efectos } \\
\text { positivos a corto plazo. } \\
\text { - El peor desempeño se } \\
\text { incrementa con la } \\
\text { gravedad de la adicción. }\end{array}$ \\
\hline $\begin{array}{l}\text { Dong et al. } \\
\text { (2015) }\end{array}$ & $\begin{array}{l}71 \text { estudiantes } \\
\text { universitarios } \\
-35 \text { AI (juegos) } \\
-36 \text { Controles }\end{array}$ & $\begin{array}{l}\text { Juegos } \\
\text { on line }\end{array}$ & IAT & $\begin{array}{l}\text { Control } \\
\text { inhibitorio }\end{array}$ & Stroop Task & MINI & t-Student & $\begin{array}{l}\text { No se encontraron } \\
\text { diferencias significativas } \\
\text { en la interferencia Stroop. }\end{array}$ & $\begin{array}{l}\text { A pesar de una mayor } \\
\text { actividad durante la tarea } \\
\text { en la corteza cingulada, los } \\
\text { AI no mostraron } \\
\text { diferencias en la capacidad } \\
\text { de control inhibitorio. }\end{array}$ \\
\hline $\begin{array}{l}\text { Yao et al., } \\
\text { (2015) }\end{array}$ & $\begin{array}{l}102 \text { estudiantes } \\
\text { universitarios } \\
\text { hombres } \\
\text { - } 60 \text { AI (juegos) } \\
-\quad 42 \text { Controles }\end{array}$ & $\begin{array}{l}\text { Juegos } \\
\text { on line }\end{array}$ & CIAS & $\begin{array}{l}\text { Toma de } \\
\text { decisiones }\end{array}$ & Cups Task & & $\begin{array}{l}\text { t-Student } \\
\text { ANOVA } \\
\text { Correlación }\end{array}$ & $\begin{array}{l}\text { El grupo IGD opta por } \\
\text { opciones más arriesgadas en } \\
\text { la condición de desventaja } \\
(p<.001) \text {. } \\
\text { Correlación directa entre la } \\
\text { puntuación en el CIAS y la } \\
\text { condición de desventaja } \\
(p<.05)^{*} \text {. }\end{array}$ & $\begin{array}{l}\text { Los adictos al juego on line: } \\
\text { - Toman decisiones más } \\
\text { arriesgadas. } \\
\text { - La mayor gravedad en la } \\
\text { adicción se relaciona con } \\
\text { decisiones menos } \\
\text { ventajosas. }\end{array}$ \\
\hline
\end{tabular}


Tabla 5 (continua).Resultados encontrados en la revisión sistemática

\begin{tabular}{|c|c|c|c|c|c|c|c|c|c|}
\hline $\begin{array}{l}\text { Referencia } \\
\text { del estudio }\end{array}$ & Muestra & $\begin{array}{l}\text { Subtipo } \\
\text { de AI }\end{array}$ & $\begin{array}{l}\text { Instrumento } \\
\text { de valoración } \\
\text { AI }\end{array}$ & $\begin{array}{l}\text { FFEE } \\
\text { evaluada }\end{array}$ & $\begin{array}{l}\text { Instrumento } \\
\text { de valoración } \\
\text { FFEE }\end{array}$ & $\begin{array}{l}\text { Otras } \\
\text { pruebas } \\
\text { aplicadas }\end{array}$ & $\begin{array}{l}\text { Análisis } \\
\text { realizados }\end{array}$ & Resultados & Conclusión \\
\hline $\begin{array}{l}\text { Balconi et } \\
\text { al., (2016) }\end{array}$ & $\begin{array}{l}28 \text { estudiantes } \\
\text { universitarios } \\
-12 \text { AI } \\
-16 \text { Controles }\end{array}$ & General & IAT & $\begin{array}{l}\text { Control } \\
\text { inhibitorio }\end{array}$ & $\begin{array}{l}\text { Go/no-GoTask } \\
\text { modificada } \\
\text { (videojuegos y } \\
\text { apuestas) }\end{array}$ & $\begin{array}{l}\text { BIS } \\
\text { BAS } \\
\text { STAI } \\
\text { BDI }\end{array}$ & $\begin{array}{l}\text { ANOVA } \\
\text { Medidas } \\
\text { repetidas } \\
\text { Post hoc }\end{array}$ & $\begin{array}{l}\text { El grupo de AI presenta un } \\
\text { TR menor en la versión } \\
\text { modificada videojuegos } \\
(p=.001) \text { y la versión } \\
\text { modificada apuestas } \\
(p=.001) .\end{array}$ & $\begin{array}{l}\text { Las personas con AI } \\
\text { muestran déficits en la } \\
\text { inhibición cuando los } \\
\text { estímulos son gratificantes } \\
\text { para el sujeto. }\end{array}$ \\
\hline $\begin{array}{l}\text { Dong y } \\
\text { Potenza } \\
\text { (2016) }\end{array}$ & $\begin{array}{l}36 \text { estudiantes } \\
\text { universitarios } \\
\text { hombres } \\
-20 \text { AI (juegos) } \\
-16 \text { Controles }\end{array}$ & $\begin{array}{l}\text { Juegos } \\
\text { on line }\end{array}$ & IAT & $\begin{array}{l}\text { Toma de } \\
\text { decisiones }\end{array}$ & Risk Task & MINI & t-Student & $\begin{array}{l}\text { Los AI (juego): } \\
\text { Durante la fase de } \\
\text { decisión toman decisiones } \\
\text { más arriesgadas en la } \\
\text { condición desventajosa } \\
(p=.039) \text {. } \\
\text { Durante la fase de apuesta } \\
\text { tienen un TR inferior tras } \\
\text { seleccionar la opción } \\
\text { desventajosa ( } p=.006) \text {. }\end{array}$ & $\begin{array}{l}\text { Las personas con adicción } \\
\text { al juego on line toman } \\
\text { decisiones sesgadas hacia } \\
\text { las recompensas a corto } \\
\text { plazo y además asumen } \\
\text { más riesgos. }\end{array}$ \\
\hline $\begin{array}{l}\text { Jeromin et } \\
\text { al., (2016) }\end{array}$ & $\begin{array}{l}51 \text { estudiantes } \\
\text { universitarios } \\
-21 \text { AI (juegos) } \\
-30 \text { Controles }\end{array}$ & $\begin{array}{l}\text { Juegos } \\
\text { on line }\end{array}$ & CIUS-WoW & $\begin{array}{l}\text { Control } \\
\text { inhibitorio }\end{array}$ & $\begin{array}{l}\text { Addiction } \\
\text { Stroop Taks }\end{array}$ & $\begin{array}{l}\text { Ishihara } \\
\text { Test } \\
\text { Visual } \\
\text { Probe }\end{array}$ & ANOVA & $\begin{array}{l}\text { Addiction Stroop: los } \\
\text { jugadores respondieron } \\
\text { más lento ante palabras } \\
\text { relacionadas con } \\
\text { ordenadores con respecto } \\
\text { a a las neutras }(p=.001) \\
\text { Visual Probe: no se } \\
\text { encontraron diferencias en } \\
\text { el TR entre imágenes } \\
\text { relacionadas con } \\
\text { ordenadores y neutras en } \\
\text { ningún grupo. }\end{array}$ & $\begin{array}{l}\text { Sesgo atencional y } \\
\text { dificultades de control de } \\
\text { interferencia con los } \\
\text { estímulos verbales } \\
\text { relacionados con los } \\
\text { ordenadores, pero no con } \\
\text { los visuales. } \\
\text { Los autores interpretan esta } \\
\text { discrepancia por la } \\
\text { facilidad de los jugadores } \\
\text { para procesar estímulos } \\
\text { visuales. }\end{array}$ \\
\hline
\end{tabular}


Tabla 6 (continua).Resultados encontrados en la revisión sistemática

\begin{tabular}{|c|c|c|c|c|c|c|c|c|c|}
\hline $\begin{array}{l}\text { Referencia } \\
\text { del estudio }\end{array}$ & Muestra & $\begin{array}{l}\text { Subtipo } \\
\text { de AI }\end{array}$ & $\begin{array}{l}\text { Instrumento } \\
\text { de valoración } \\
\text { AI }\end{array}$ & $\begin{array}{l}\text { FFEE } \\
\text { evaluada }\end{array}$ & $\begin{array}{l}\text { Instrumento } \\
\text { de valoración } \\
\text { FFEE }\end{array}$ & $\begin{array}{l}\text { Otras } \\
\text { pruebas } \\
\text { aplicadas }\end{array}$ & $\begin{array}{l}\text { Análisis } \\
\text { realizados }\end{array}$ & Resultados & Conclusión \\
\hline $\begin{array}{l}\text { Nie et al., } \\
\text { (2016) }\end{array}$ & $\begin{array}{l}16 \text { estudiantes } \\
\text { universitarios } \\
-\quad 53 \text { AI severa } \\
-\quad 95 \text { AI moderada } \\
-\quad 168 \text { Controles }\end{array}$ & General & CIAS-R & Fluidez verbal & $\begin{array}{l}\text { Tarea de } \\
\text { Fluidez Verbal }\end{array}$ & $\begin{array}{l}\text { ZSDS } \\
\text { RSES }\end{array}$ & $\begin{array}{l}\text { ANOVA } \\
\text { Post-hoc }\end{array}$ & $\begin{array}{l}\text { Los grupos AI-severa } \\
\text { ejecutan peor la tarea de } \\
\text { fluidez verbal semántica } \\
\text { que los AI moderada y los } \\
\text { no adictos ( } p=.001) \text {. No } \\
\text { existen diferencias en la } \\
\text { tarea de fluidez verbal } \\
\text { fonológica entre grupos. }\end{array}$ & $\begin{array}{l}\text { La alteración en la fluidez } \\
\text { verbal semántica puede ser } \\
\text { un marcador estable útil a } \\
\text { la hora de identificar la } \\
\text { transición entre la adicción } \\
\text { moderada y la adicción } \\
\text { severa. }\end{array}$ \\
\hline $\begin{array}{l}\text { Zhang et al., } \\
\text { (2016) }\end{array}$ & $\begin{array}{l}40 \text { estudiantes } \\
\text { universitarios } \\
\text { - } 19 \text { AI (Juegos) } \\
\text { - } 21 \text { Controles }\end{array}$ & $\begin{array}{l}\text { Juegos } \\
\text { on line }\end{array}$ & IAT & $\begin{array}{l}\text { Control } \\
\text { inhibitorio }\end{array}$ & $\begin{array}{l}\text { Addiction } \\
\text { Stroop Task }\end{array}$ & & $\begin{array}{l}\text { ANOVA } \\
\text { Medidas } \\
\text { repetidas }\end{array}$ & $\begin{array}{l}\text { No hubo efecto de } \\
\text { interacción entre el grupo } \\
\text { (adictos/no adictos) y tipo } \\
\text { de EE (neutro/relacionad } \\
\text { con juego on line). }\end{array}$ & $\begin{array}{l}\text { Loa adictos no muestran } \\
\text { sesgo atencional ni } \\
\text { dificultades de inhibición } \\
\text { ante estímulos } \\
\text { relacionados con la } \\
\text { adicción. }\end{array}$ \\
\hline $\begin{array}{l}\text { Balconi et } \\
\text { al., (2017a) }\end{array}$ & $\begin{array}{l}24 \text { estudiantes } \\
\text { universitarios } \\
-\quad 12 \mathrm{AI} \\
-\quad 12 \text { Controles }\end{array}$ & General & IAT & $\begin{array}{l}\text { Control } \\
\text { inhibitorio }\end{array}$ & $\begin{array}{l}\text { Go/no-Go Task } \\
\text { modificada } \\
\text { EE videojuegos } \\
\text { EE apuestas } \\
\text { EE neutros }\end{array}$ & $\begin{array}{l}\text { BIS } \\
\text { BAS }\end{array}$ & $\begin{array}{l}\text { ANOVA } \\
\text { medidas } \\
\text { repetidas } \\
\text { Post hoc }\end{array}$ & $\begin{array}{l}\text { En la condición no-Go el } \\
\text { grupo AI tiene una mejor } \\
\text { ejecución: menos errores } \\
\text { para los EE relacionados } \\
\text { con videojuegos } \\
(p=0.001) \text { y apuestas } \\
(p=0.001) \text {, así como TR } \\
\text { menores tanto para los EE } \\
\text { de videojuegos ( } p=0.001) \\
\text { como de apuestas } \\
(p=0.001) .\end{array}$ & $\begin{array}{l}\text { Existencia de un sesgo } \\
\text { atencional hacia estímulos } \\
\text { que potencialmente pueden } \\
\text { implicar una recompensa, } \\
\text { caracterizado por una } \\
\text { mayor eficacia cognitiva } \\
\text { cuando se requiere mayor } \\
\text { control. }\end{array}$ \\
\hline
\end{tabular}


Tabla 7 (continua).Resultados encontrados en la revisión sistemática

\begin{tabular}{|c|c|c|c|c|c|c|c|c|c|}
\hline $\begin{array}{l}\text { Referencia } \\
\text { del estudio }\end{array}$ & Muestra & $\begin{array}{l}\text { Subtipo } \\
\text { de AI }\end{array}$ & $\begin{array}{l}\text { Instrumento } \\
\text { de valoración } \\
\text { AI }\end{array}$ & $\begin{array}{l}\text { FFEE } \\
\text { evaluada }\end{array}$ & $\begin{array}{l}\text { Instrumento } \\
\text { de valoración } \\
\text { FFEE }\end{array}$ & $\begin{array}{l}\text { Otras } \\
\text { pruebas } \\
\text { aplicadas }\end{array}$ & $\begin{array}{l}\text { Análisis } \\
\text { realizados }\end{array}$ & Resultados & Conclusión \\
\hline $\begin{array}{l}\text { Balconi et } \\
\text { al., (2017b) }\end{array}$ & $\begin{array}{l}25 \text { estudiantes } \\
\text { universitarios } \\
-\quad 12 \mathrm{AI} \\
-\quad 13 \text { Controles }\end{array}$ & General & IAT & $\begin{array}{l}\text { Control } \\
\text { inhibitorio }\end{array}$ & $\begin{array}{l}\text { Go/no-Go Task } \\
\text { modificada } \\
\text { EE videojuegos } \\
\text { EE apuestas } \\
\text { EE neutros }\end{array}$ & $\begin{array}{l}\text { STAI } \\
\text { BDI }\end{array}$ & $\begin{array}{l}\text { ANOVA } \\
\text { medidas } \\
\text { repetidas } \\
\text { Post hoc }\end{array}$ & $\begin{array}{l}\text { Mismos resultados estudio } \\
\text { anterior. }\end{array}$ & $\begin{array}{l}\text { La mayor sensibilidad a la } \\
\text { recompensa puede ser una } \\
\text { variable crucial en el } \\
\text { desarrollo de la adicción. }\end{array}$ \\
\hline $\begin{array}{l}\text { Dong, et al. } \\
\text { (2017) }\end{array}$ & $\begin{array}{l}58 \text { estudiantes } \\
\text { universitarios } \\
\text { (hombres) } \\
-18 \text { AI (juegos) } \\
-21 \text { jugadores no } \\
\text { AI } \\
-19 \text { Controles }\end{array}$ & $\begin{array}{l}\text { Juegos } \\
\text { on line }\end{array}$ & IAT & $\begin{array}{l}\text { Control } \\
\text { inhibitorio }\end{array}$ & $\begin{array}{l}\text { Stroop Task } \\
\text { Guessing Task }\end{array}$ & & ANOVA & $\begin{array}{l}\text { El grupo de adictos a los } \\
\text { juegos on line mostró } \\
\text { mayor interferencia Stroop } \\
\text { (mayor TR) que los } \\
\text { jugadores no adictos } \\
(p=.005) \text { y que el grupo } \\
\text { control ( } p=.048) \text {. } \\
\text { No hay diferencias entre } \\
\text { grupos en la ejecución de } \\
\text { la Guessing Task. }\end{array}$ & $\begin{array}{l}\text { La adicción a los juegos on } \\
\text { line se relaciona con un } \\
\text { peor control inhibitorio. }\end{array}$ \\
\hline $\begin{array}{l}\text { He et al., } \\
\text { (2017) }\end{array}$ & $\begin{array}{l}32 \text { estudiantes } \\
\text { universitarios } \\
\text { hombres } \\
\text { - } 16 \text { AI } \\
\text { - } 16 \text { Controles }\end{array}$ & General & IADC & $\begin{array}{l}\text { Toma de } \\
\text { decisiones }\end{array}$ & $\begin{array}{l}\text { Force-choice } \\
\text { Gambling Task }\end{array}$ & & t-Student & $\begin{array}{l}\text { El grupo AI tiende a tomar } \\
\text { decisiones más arriesgadas } \\
(p<.001)^{*} \text {. }\end{array}$ & $\begin{array}{l}\text { La adicción a internet se } \\
\text { relaciona con una } \\
\text { alteración en la toma de } \\
\text { decisiones. }\end{array}$ \\
\hline $\begin{array}{l}\text { Jiang et al., } \\
\text { (2017) }\end{array}$ & $\begin{array}{l}98 \text { estudiantes } \\
\text { universitarios } \\
-\quad 27 \text { (s- OSA) } \\
-\quad 43 \text { (m-OSA) } \\
-\quad 28 \text { (1- OSA) }\end{array}$ & $\begin{array}{l}\text { Compras } \\
\text { on line }\end{array}$ & OSAS & $\begin{array}{l}\text { Control } \\
\text { inhibitorio } \\
\text { Control } \\
\text { atencional }\end{array}$ & $\begin{array}{l}\text { Stroop Task } \\
\text { modificado } \\
\text { Dot Probe Task }\end{array}$ & $\begin{array}{l}\text { BIS } \\
\text { BAS } \\
\text { SCS }\end{array}$ & $\begin{array}{l}\text { ANOVA } \\
\text { Correlación }\end{array}$ & $\begin{array}{l}\text { En la tarea Stroop el grupo s- } \\
\text { OSA fue más rápido ante las } \\
\text { palabras neutras que antes las } \\
\text { relacionadas con compras on } \\
\text { line (p <.001), En los grupos } \\
\text { m-OSA y M-OSA no hubo } \\
\text { diferencias en función del } \\
\text { tipo de palabra. } \\
\text { No hubo diferencias en la Dot } \\
\text { Probe Task, ni relación entre } \\
\text { los resultados de las dos } \\
\text { tareas. }\end{array}$ & $\begin{array}{l}\text { Las personas con adicción } \\
\text { a las compras on line } \\
\text { tienen un sesgo atencional } \\
\text { hacia EE asociados con la } \\
\text { adicción, caracterizado por } \\
\text { un menor control } \\
\text { inhibitorio. }\end{array}$ \\
\hline
\end{tabular}


Tabla 8 (continua).Resultados encontrados en la revisión sistemática

\begin{tabular}{|c|c|c|c|c|c|c|c|c|c|}
\hline $\begin{array}{l}\text { Referencia } \\
\text { del estudio }\end{array}$ & Muestra & $\begin{array}{l}\text { Subtipo } \\
\text { de AI }\end{array}$ & $\begin{array}{l}\text { Instrumento } \\
\text { de valoración } \\
\text { AI }\end{array}$ & $\begin{array}{l}\text { FFEE } \\
\text { evaluada }\end{array}$ & $\begin{array}{l}\text { Instrumento } \\
\text { de valoración } \\
\text { FFEE } \\
\end{array}$ & $\begin{array}{l}\text { Otras } \\
\text { pruebas } \\
\text { aplicadas } \\
\end{array}$ & $\begin{array}{l}\text { Análisis } \\
\text { realizados }\end{array}$ & Resultados & Conclusión \\
\hline $\begin{array}{l}\text { Wang et al., } \\
\text { (2017a) }\end{array}$ & $\begin{array}{l}39 \text { estudiantes } \\
\text { universitarios } \\
\text { hombres } \\
\text { - } 18 \text { AI (juegos) } \\
\text { - } 21 \text { Controles }\end{array}$ & $\begin{array}{l}\text { Juegos } \\
\text { on line }\end{array}$ & IAT & $\begin{array}{l}\text { Toma de } \\
\text { decisiones }\end{array}$ & $\begin{array}{l}\text { Delay } \\
\text { Discounting } \\
\text { Task }\end{array}$ & MINI & $\begin{array}{l}\text { t-Student } \\
\text { Correlación }\end{array}$ & $\begin{array}{l}\text { El grupo de adictos accede } \\
\text { de forma más rápida al } \\
\text { descuento }(p=.05) \text {. Existe } \\
\text { correlación positiva entre } \\
\text { el TR y la tasa de demora } \\
\text { en el descuento }(p=.03) \text {. }\end{array}$ & $\begin{array}{l}\text { La adicción al juego on } \\
\text { line se relaciona con una } \\
\text { toma de decisiones más } \\
\text { arriesgada. }\end{array}$ \\
\hline $\begin{array}{l}\text { Wang et al., } \\
\text { (2017b) }\end{array}$ & $\begin{array}{l}63 \text { estudiantes } \\
\text { universitarios } \\
\text { hombres } \\
-20 \text { AI (juegos) } \\
-23 \text { jugadores no } \\
\text { AI } \\
-20 \text { Controles }\end{array}$ & $\begin{array}{l}\text { Juegos } \\
\text { on line }\end{array}$ & $\begin{array}{l}\text { IAT } \\
\text { Criterios } \\
\text { DSM-5 }\end{array}$ & $\begin{array}{l}\text { Toma de } \\
\text { decisiones }\end{array}$ & $\begin{array}{l}\text { Delay } \\
\text { Discounting } \\
\text { Task } \\
\text { Probability } \\
\text { Discounting } \\
\text { Task }\end{array}$ & & $\begin{array}{l}\text { ANOVA } \\
\text { Correlación }\end{array}$ & $\begin{array}{l}\text { El grupo de adictos accede } \\
\text { de forma más rápida al } \\
\text { descuento }(p=.03) \text { y } \\
\text { muestra mayor tendencia } \\
\text { al riesgo }(p<.05) \text { que los } \\
\text { otros dos grupos. } \\
\text { Correlación entre la } \\
\text { velocidad de descuento y } \\
\text { la tendencia al riesgo } \\
p<.001) \text {. }\end{array}$ & $\begin{array}{l}\text { Las personas con adicción } \\
\text { los juegos on line } \\
\text { presentan: } \\
\text { - Déficit en la inhibición } \\
\text { de respuestas. } \\
\text { - Toma de decisiones más } \\
\text { arriesgada. }\end{array}$ \\
\hline $\begin{array}{l}\text { Wang et al., } \\
(2017 \mathrm{c})\end{array}$ & $\begin{array}{l}39 \text { estudiantes } \\
\text { universitarios } \\
-18 \text { AI juegos) } \\
-21 \text { controles }\end{array}$ & $\begin{array}{l}\text { Juegos } \\
\text { on line }\end{array}$ & IAT & $\begin{array}{l}\text { Toma de } \\
\text { decisiones }\end{array}$ & $\begin{array}{l}\text { Delay } \\
\text { Discounting } \\
\text { Task }\end{array}$ & MINI & $\begin{array}{l}\text { t-Student } \\
\text { Correlación }\end{array}$ & $\begin{array}{l}\text { El grupo de adictos accede } \\
\text { de forma más rápida al } \\
\text { descuento }(p=.05) \\
\text { La puntuación del IAT } \\
\text { correlaciona de forma } \\
\text { directa con la velocidad de } \\
\text { descuento }(p<.05)^{*} \text {. }\end{array}$ & $\begin{array}{l}\text { Los sujetos adictos al } \\
\text { juego on line muestran } \\
\text { menor capacidad de } \\
\text { inhibición que los no } \\
\text { adictos. } \\
\text { A mayor severidad de la } \\
\text { adicción mayor } \\
\text { impulsividad en la toma } \\
\text { de decisiones. }\end{array}$ \\
\hline
\end{tabular}


Tabla 9 (continua).Resultados encontrados en la revisión sistemática

\begin{tabular}{|c|c|c|c|c|c|c|c|c|c|}
\hline $\begin{array}{l}\text { Referencia } \\
\text { del estudio }\end{array}$ & Muestra & $\begin{array}{l}\text { Subtipo } \\
\text { de AI }\end{array}$ & $\begin{array}{l}\text { Instrumento } \\
\text { de valoración } \\
\text { AI }\end{array}$ & $\begin{array}{l}\text { FFEE } \\
\text { evaluada }\end{array}$ & $\begin{array}{l}\text { Instrumento } \\
\text { de valoración } \\
\text { FFEE }\end{array}$ & $\begin{array}{l}\text { Otras } \\
\text { pruebas } \\
\text { aplicadas }\end{array}$ & $\begin{array}{l}\text { Análisis } \\
\text { realizados }\end{array}$ & Resultados & Conclusión \\
\hline $\begin{array}{l}\text { Tekin et al., } \\
\text { (2018) }\end{array}$ & $\begin{array}{l}59 \text { estudiantes } \\
\text { universitarios } \\
-30 \mathrm{AI} \\
-29 \text { Controles }\end{array}$ & General & IAS & $\begin{array}{l}\text { Control } \\
\text { inhibitorio } \\
\text { Flexibilidad } \\
\text { cognitiva }\end{array}$ & $\begin{array}{l}\text { Stroop Task } \\
\text { Trail Making } \\
\text { Test }\end{array}$ & BDI & $\begin{array}{l}\text { U Mann- } \\
\text { Whitney }\end{array}$ & $\begin{array}{l}\text { El grupo AI fue más lento } \\
\text { en la condición color e } \\
\text { interferencia }(p<.01) \text {. } \\
\text { En el TMT-B los AI } \\
\text { cometieron más errores } \\
(p<.01) \text { y tardaron más } \\
\text { tiempo en completar la } \\
\text { tarea }(p=.005) \text {. }\end{array}$ & $\begin{array}{l}\text { Las personas con AI se } \\
\text { caracterizan por } \\
\text { alteraciones cognitivas a } \\
\text { nivel atencional: peor } \\
\text { atención sostenida y } \\
\text { selectiva y déficit en el } \\
\text { control inhibitorio y en la } \\
\text { flexibilidad cognitiva. }\end{array}$ \\
\hline
\end{tabular}

Nota: $\mathrm{AI}=$ Adicción a internet; FFEE= Funciones ejecutivas; CAI= Cuestionario de Adicción a Internet; Test; TPQ=Tridimensional Personality Questionnaire; DCIA-C= Diagnostic Criteria for Internet Addiction for College Students; IGT=Iowa Gambling Task; STAI= State-Trait Anxiety Inventory; BDI= Beck Depression Inventory; IIADC= Internet Addiction Disorder Criteria; sOSA= severe Online Shopping Addiction; 1-OSA= low Online Shopping Addiction ; m-OSA= mild Online Shopping Addiction; OSA= Online Shopping Addiction; BIS= Behavioral Inhibitory System; BAS= Behavioral Activation System; SCS= Self-Control Scale; OSAS= Online Shopping Addiction

Scale; MINI= Mini International Neuropsychiatric Inventory. 
Los 30 artículos empíricos incluidos en esta revisión incluyen un total de 1830 participantes, con una gran variabilidad en el $n$ de la muestra, que oscila entre los 216 sujetos del trabajo de Ko et al., (2010) y los 17 participantes del estudio de Dong et al., (2011). En cuanto al género, se incluyeron a 587 mujeres y 1243 hombres, aunque en 15 de los trabajos la muestra estuvo constituida únicamente por hombres. Los estudios que incluyen ambos géneros no siempre utilizan muestras homogéneas, superando en ocasiones la proporción de hombres a la de mujeres (Sun et al., 2009; Ko et al., 2010), mientras que en otras se observa el patrón contrario (Nie et al., 2016; Saville et al., 2010). En cuanto al subtipo de adicción a internet, 12 estudios tratan el trastorno de forma general, sin distinguir aplicación objeto del uso problemático. 17 trabajos han estudiado únicamente adictos al juego online o gaming, y 1 estudio se centra en las compras patológicas online. No se ha encontrado ningún artículo que estudiase de forma específica la adicción a contenidos pornográficos ni a las apuestas on line.

Aunque un número importante de trabajos utiliza el Internet Addiction Test (IAT; Young, 1998) para la evaluación del uso problemático o adicción a internet, destaca la variabilidad de instrumentos utilizados. A continuación (tabla 2) se describe brevemente las herramientas utilizadas en los estudios de esta revisión.

Tabla 2. Instrumentos utilizados para valorar la adicción a internet

\begin{tabular}{|c|c|c|}
\hline Instrumento & Descripción & Estudios \\
\hline $\begin{array}{l}\text { Internet Addiction Test, IAT } \\
\text { (Young, 1998) }\end{array}$ & $\begin{array}{l}\text { Cuestionario realizado adaptando los criterios del DSM- } \\
\text { IV para juego patológico. Conformado } 8 \text { ítems } \\
\text { dicotómicos evalúa: preocupación, tolerancia, síntomas } \\
\text { de abstinencia, pérdida de control, pérdida del interés por } \\
\text { otras actividades, preocupación del entorno y uso por } \\
\text { evitación de la disforia. Un número de } 5 \text { o más respuestas } \\
\text { afirmativas ("sí") se considera adicción a Internet. } \\
\text { Ningún estudio reporta datos de consistencia interna. }\end{array}$ & $\begin{array}{l}\text { Sun et al., (2009); Dong } \\
\text { et al., (2010); Saville et } \\
\text { al., (2010); Dong et al., } \\
\text { (2011). }\end{array}$ \\
\hline $\begin{array}{l}\text { Internet Addiction Scale, IAS, } \\
\text { (Griffith, 1998) }\end{array}$ & $\begin{array}{l}\text { Escala tipo Likert compuesta por } 28 \text { ítems. Puntuaciones } \\
\text { mayores a } 81 \text { se considera indicativo de adicción a } \\
\text { Internet. La consistencia interna (alfa de Cronbach) es } \\
\text { igual a } \alpha=.94 \text {. }\end{array}$ & Tekin et al., (2018). \\
\hline $\begin{array}{l}\text { Chen Internet Addiction Scale, } \\
\text { CIAS, (Chen et al., 2003) }\end{array}$ & $\begin{array}{l}\text { Cuestionario conformado por } 26 \text { ítems tipo Likert } \\
\text { agrupados en } 5 \text { subescalas: uso compulsivo, síntomas de } \\
\text { abstinencia, tolerancia, problemas en relaciones } \\
\text { interpersonales y administración del tiempo. Ningún } \\
\text { estudio reporta datos de consistencia interna. }\end{array}$ & $\begin{array}{l}\text { Yao et al., (2014); Yao } \\
\text { et al., (2015). }\end{array}$ \\
\hline $\begin{array}{l}\text { Online Shopping Addiction } \\
\text { Scale, OSAS (Xu, 2007) }\end{array}$ & $\begin{array}{l}\text { Escala de } 22 \text { ítems tipo Likert que evalúan: alteración de } \\
\text { la funcionalidad, consumo excesivo, síntomas de } \\
\text { abstinencia y placer. La consistencia interna (alfa de } \\
\text { Cronbach) es igual a } \alpha=.90\end{array}$ & Jiang et al., (2017) \\
\hline
\end{tabular}


Tabla 2 (Continua). Instrumentos utilizados para valorar la adicción a internet

\begin{tabular}{|c|c|c|}
\hline Instrumento & Descripción & Estudios \\
\hline $\begin{array}{l}\text { Diagnostic Criteria for } \\
\text { Internet Addiction for } \\
\text { College Students, } \\
\text { DCIA-C, (Ko, Chen, Yang, } \\
\text { Lin y Yen, 2009) }\end{array}$ & $\begin{array}{l}\text { Conformado por } 3 \text { criterios. El criterio A evalúa los } \\
\text { síntomas característicos (preocupación, pérdida de } \\
\text { control sobre impulsos, más uso del planeado, } \\
\text { tolerancia, síntomas de abstinencia, pérdida de } \\
\text { control, tiempo excesivo de uso y alteración en la } \\
\text { toma de decisiones). El criterio B evalúa las } \\
\text { alteraciones secundarias al uso de Internet, mientras } \\
\text { que el criterio C se corresponde con los criterios de } \\
\text { exclusión. No se especifica ni el número de ítems ni } \\
\text { la consistencia interna del cuestionario. }\end{array}$ & Ko et al., (2010). \\
\hline $\begin{array}{l}\text { Internet Addiction Test, IAT } \\
\text { (Young, 2009) }\end{array}$ & $\begin{array}{l}\text { Cuestionario conformado por } 20 \text { ítems tipo Likert, } \\
\text { que evalúan } 5 \text { dimensiones: dependencia } \\
\text { psicológica, síntomas de abstinencia, problemas en } \\
\text { colegio, trabajo, familia o sueño y pérdida de } \\
\text { control del tiempo de uso. Puntuaciones mayores a } \\
50 \text { se corresponden a un uso frecuente, por encima } \\
\text { de } 80 \text { se considera adicción a Internet. La } \\
\text { consistencia interna (alfa de Cronbach) varía entre } \\
\alpha=.81 \text { y } \alpha=.969 \text {. }\end{array}$ & $\begin{array}{l}\text { Dong et al., (2014); } \\
\text { Xing et al., (2014); } \\
\text { Dong et al., (2014); } \\
\text { Balconi et al., (2016); } \\
\text { Dong et al., (2016); } \\
\text { Zhang et al., (2016); } \\
\text { Balconi et al., (2017a, } \\
\text { b). Dong et al., } \\
\text { (2017); Wang et al., } \\
\text { (2017a,b,c,d). }\end{array}$ \\
\hline
\end{tabular}

Internet Addiction

Diagnostic Criteria, IADC, (Tao et al., 2010)

Videogame Addiction Test, VAT, (van Rooij,

Schoenmakers, Van den Eijnden, Vermulst y van de Mheen, 2012)

Compulsive Internet Use Scale World of Worldcraft specific, CIUS-WoW

(Barke, Nyenhuis, Voigots, Gehrke y Kröner-Herwig, 2013)

Media Usage Questionnaire, MUQ, (Bailey et al., 2013)

Chen Internet Addiction Scale Revised, CIAS-R (Mak et al., 2014)
Consta de 7 ítems que evalúan: preocupación, síntomas de abstinencia, tolerancia, pérdida de control, reiteración del uso a pesar de las consecuencias negativas, pérdida de interés por otras actividades y uso para evitar la disforia. No se indica la consistencia interna.

Adaptado del CIUS (Meekerk et al., 2009) consta de 14 ítems tipo Likert. La consistencia interna del test (alfa de Cronbach) es igual a $\alpha=.93$.

Escala tipo Likert. Puntuaciones superiores a 25 se consideran indicativas de adicción a Internet. La consistencia interna (alfa de Cronbach) es igual a $\alpha=.86$.

Cuestionario de uso de internet, conformado por 3 ítems en los cuales los sujetos indican el número de horas dedicadas a videojuegos, los juegos más utilizados y cuánto tiempo dedica al preferido.

Escala tipo Likert 27 ítems. Puntuaciones superiores a 63 se consideran como adicción a Internet. La consistencia interna (alfa de Cronbach) varía entre $\alpha=.79$ y $\alpha=.93$. 
Dentro de las funciones ejecutivas, los estudios revisados se han centrado principalmente en el control inhibitorio y en la toma de decisiones. Se encuentra disparidad de resultados. Para valorar el control inhibitorio, se ha utilizado el paradigma Go/no-Go Task, así como la tarea Stroop, original y modificada. Muchos trabajos han encontrado una afectación del control inhibitorio en relación con la adicción a internet (Balconi et al., 2016; Dong, et al. 2017; Jeromin et al., 2016; Jiang et al., 2017; Littel et al., 2012; Tekin et al., 2018; Wang et al., 2017b; Xing, et al., 2014; Yao et al., 2014), o, de forma coherente, mayor impulsividad (Saville et al., 2010) y menor flexibilidad cognitiva (Tekin et al., 2018). En contraste, en algunos estudios no se da tal alteración (Dong et al., 2010; Dong et al., 2014; Dong et al., 2015; Sun et al 2009; Wang et al., 2017d; Zhang et al., 2016), encontrándose en algunos casos un mejor control inhibitorio en los sujetos adictos para estímulos con carácter de recompensa (Balconi et al., 2017a; Balconi et al., 2017b). En uno de los estudios la afectación o no del control inhibitorio en la adicción al juego dependió del tipo de juego (disparos /estrategia) (Bailey et al., 2013).

En cuanto a la toma de decisiones, se han utilizado diferentes pruebas de apuestas (Iowa Gambling Task, Modified Gambling Task; Forced-Choice Gambling Task), pruebas de descuento (Delay Discount Task, Temporal Discount, Probability Discounting), y otro tipo de pruebas que implican alguna forma de recompensa (Game of Dice, Cups Task). Se ha encontrado una alteración en la toma de decisiones afectada en algunos estudios (Dong et al., 2016; He et al., 2017; Pawllikoswki et al., 2011; Sun et al., 2009; Wang et al., 2017b; Yao et al., 2014; Yao et al., 2015). Otros trabajos encuentran resultados contrapuestos: en uno de ellos no se encuentra déficit en la toma de decisiones (Ko et al., 2010), en otro la alteración o no depende del tipo de juego (Bailey et al., 2013), o está relacionada con el comportamiento cooperativo (Su et al., 2018). un estudio valoró la fluidez verbal encontrando una afectación de la fluidez semántica en relación con la adicción a internet, pero no de la fluidez fonológica (Nie et al., 2016). La flexibilidad cognitiva se encuentra afectada en el único trabajo que la evalúa con una tarea específica (TMT B) (Tekin et al., 2018). Por otra parte, un estudio encontró mayor rendimiento en situaciones multitareas en adictos al juego online, aunque únicamente en tareas ecológicas, frente a tareas artificiales de laboratorio (Chen et al., 2018). La gestión y el desempeño en contextos multitarea guarda relación directa con la flexibilidad y el control inhibitorio (Rothbart y Posner, 2015). No se han encontrado estudios que aborden directamente la memoria de trabajo en relación con el uso excesivo de internet. 


\section{Discusión}

El objetivo del presente estudio era identificar variables cognitivas de riesgo para el desarrollo de adicción a internet entre la población universitaria. Los resultados encontrados sugieren de forma general la alteración de dos componentes ejecutivos de gran importancia en el desarrollo de adicciones a internet, estos son, el control inhibitorio y la toma de decisiones. Sin embargo, las conclusiones de los distintos estudios no han resultado siempre convergentes. Muchos trabajos han relacionado un déficit en el control inhibitorio con un uso abusivo de la web, aunque gran parte de estos estudios se refieren de forma exclusiva a la adicción al juego online o gaming. Los resultados sobre control inhibitorio obtenidos con jugadores online deben tomarse con cautela, ya que esta aplicación podría tener características diferenciales (competitividad, velocidad de respuesta) que parecen no compartir otro tipo de aplicaciones, como la visión de contenidos pornográficos o apuestas online. En este sentido, Bailey et al. (2013) encuentran que la alteración del control inhibitorio es exclusiva de los adictos a juegos de disparos, y no se dan en jugadores de juegos de estrategia, que muestran un control cognitivo similar a los controles. Otro dato interesante es que en algunos casos el problema de control inhibitorio no es general sino específico para algunos tipos de estímulos. Así, en el caso de la adicción a compras online, Jiang et al., (2017) encuentran que el déficit inhibitorio únicamente ocurre para estímulos relacionados con la adicción, y no para otro tipo de material. Jeromin et al., (2016) concluyen que el peor control de los jugadores adictos es únicamente para estímulos verbales, y no visuales. Y como contraste, algunos trabajos encuentran mejor inhibitorio control en adictos a internet ante estímulos que podrían suponer una gratificación para el sujeto, algo que para los autores es indicativo de que una mayor sensibilidad a la recompensa constituye un factor de vulnerabilidad para el desarrollo de la adicción (Balconi et al., 2017a; Balconi et al., 2017b). Este mejor control inhibitorio también se deduce del estudio realizado con Chen et al., (2018) con adictos al juego online, cuyos resultados indican mejor rendimiento en situaciones multitarea, aunque únicamente en tareas ecológicas y no en diseños experimentales de laboratorio. En su conjunto, estos resultados permiten concluir sobre la existencia de una relación entre control inhibitorio y adicción a internet, aunque es necesario profundizar acerca de la naturaleza de esta relación en las diferentes aplicaciones o subtipos de internet, y analizar el papel de variables como el tipo de estímulo o de tarea. 
La mayor parte de los trabajos que han estudiado la toma de decisiones en relación con la adicción a internet muestra también alteraciones, caracterizadas en general por una tendencia a tomar decisiones más arriesgadas. Nuevamente los participantes de muchos de estos trabajos son adictos al juego online o gaming. De igual forma que ocurría con el control inhibitorio, esta alteración parece específica de los juegos de disparos frente a los juegos de estrategia (Bailey et al., 2013). Un estudio indica que este patrón es consecuencia de un cambio de estrategia más lento cuando se modifican las condiciones de la tarea (Sun et al., 2009), y en otro la alteración de la toma de decisiones ocurrió en situaciones de comportamiento cooperativo (Su et al., 2018). Para Ko et al. (2014), el desempeño general en tareas de toma de decisiones no es peor en los adictos, aunque muestran un perfil diferente que los controles, y tienden a decisiones que buscan la novedad. La disparidad de resultados también puede deberse a que las tareas de toma de decisiones pueden estar influidas por variables ambientales (Cohen y Aston-Jones, 2005; Ernst y Paulus, 2005) y relacionada con variables cognitivas como la falacia del jugador (Xue, Lu, Levin y Bechara, 2011). En definitiva, aunque los resultados sugieren una tendencia hacia decisiones más arriesgadas en los adictos a internet, los procesos responsables de esta tendencia (búsqueda de novedad, aprendizaje más lento de la nueva situación tras cambio de set) deberían ser objeto de una investigación en profundidad, con el fin de desarrollar e implementar estrategias eficaces para la prevención de este tipo de adicciones.

Uno de los trabajos revisados muestra alteraciones en la fluidez verbal en relación con la adicción a internet, en concreto una disminución de la fluidez semántica (no fonológica) (Nie et al., 2016). Las tareas de fluidez verbal se incluyen habitualmente en la evaluación neuropsicológica, tanto en el ámbito clínico como en investigación, y no únicamente como medida del procesamiento del lenguaje (Whiteside et al., 2016), sino con frecuencia como prueba de control ejecutivo (Aita et al., 2016; Fitzpatrick, Gilbert y Serpell, 2013; Henry y Crawford, 2004). Considerando la gran cantidad de participantes de este estudio (361), parece relevante tener en cuenta estos resultados a la hora de describir perfiles cognitivos en relación con la adicción a internet, y considerar que las tareas de fluidez verbal, además del componente lingüístico, requieren de la integridad del componente ejecutivo. 
En relación a la flexibilidad cognitiva, los resultados de los dos estudios que abordan este proceso no son convergentes. Un estudio (Tekin et al., 2018) encuentra una alteración en tareas de flexibilidad en relación al uso abusivo de internet, pero los resultados del estudio de Chen et al. (2018) en contextos multitarea indican diferente ejecución en función de la naturaleza de la tarea: el peor desempeño se daba únicamente en las tareas de laboratorio, y no en contextos cotidianos. Sería necesario profundizar en este aspecto, dada la importancia de la flexibilidad cuando en situaciones que requieren gestionar diferentes actividades de forma simultánea, tan frecuentes en la vida cotidiana. Por último, llama la atención la falta de estudios que aborden directamente la memoria de trabajo en esta revisión, y parece necesario incluir esta función en relación con el uso excesivo de internet.

Una limitación importante al intentar sacar conclusiones globales de la presente revisión se refiere a los instrumentos empleados en la identificación de la adicción a internet. Además de las limitaciones propias de los autoinformes (como la deseabilidad social), hay que destacar la disparidad de tests de evaluación de la adicción a internet utilizados. Algunos de los cuestionarios utilizados (como el Internet Addiction Test (IAT), o el Internet Addiction Scale (IAS) han demostrado su consistencia y fiabilidad y proponen un punto de corte para el diagnóstico de trastorno por adicción a internet, que permite agrupar a los participantes de forma clara. En contraste, se utilizan otras herramientas como el Diagnostic Criteria for Internet Addiction for College Students (DCIA-C), o el Media Usage Questionnaire (MUQ), desarrollada por los mismos autores, sin datos de fiabilidad y que no especifican punto de corte, de forma que una puntuación alta en estos cuestionarios podría indicar un uso frecuente (incluso excesivo) de la web, pero que no comporte las características de la conducta adictiva. Este es el caso del estudio de Bailey et al., (2013), en el que participaron 149 sujetos evaluados a través de un cuestionario (MUG) conformado por 3 ítems que valoran el número de horas al juego on line, los juegos más utilizados y cuánto tiempo dedica al preferido.

Asimismo, el rendimiento de los participantes en las tareas propuestas para valorar las funciones ejecutivas evaluadas podría estar influenciado por el entrenamiento de los sujetos en el uso frecuente de juegos online, donde pueden haberse enfrentado en muchas ocasiones a situaciones similares a las condiciones experimentales o a las tareas propuestas. Sería interesante tener en cuenta estas dos limitaciones a la hora de diseñar futuras investigaciones. 
Aunque el estudio de las diferencias por género no era objetivo del presente trabajo, en general la proporción de mujeres evaluadas fue mucho menor, y en la mitad de los trabajos los participantes fueron hombres exclusivamente. Estudios previos han mostrado que los chicos son más propensos al uso problemático de la web y que preferentemente se implican en actividades de juegos o gaming y en visualizar contenidos pornográficos, mientras que las chicas pasan más tiempo en las redes sociales (Dufour et al., 2016). Dado que una serie de estudios (Adan, 2012; White et al., 2011) indican que los hombres presentan con mayor frecuencia conductas impulsivas, decisiones arriesgadas y consumo de sustancias con respecto a las mujeres, parece interesante incluir la variable género en estudios futuros sobre la relación entre funciones ejecutivas y adicciones a internet.

En definitiva, aunque los trabajos empíricos sugieren la implicación de las funciones ejecutivas (en concreto el control inhibitorio, la toma de decisiones y la fluidez verbal) en las adicciones a internet, los resultados obtenidos no permiten establecer un perfil de riesgo claro para el desarrollo de adicciones a internet entre la población universitaria. Parece necesario profundizar en la naturaleza de esta relación diferenciando distintos subtipos de adicción, porque las características diferenciales de cada aplicación dificultan la comparación de resultados. Además, es importante incluir el control de determinadas variables, como el género, la naturaleza de la tarea y el tipo de estímulo para sacar conclusiones que permitan desarrollar estrategias preventivas y de tratamiento eficaces aplicables a esta población vulnerable al desarrollo de adicción a internet.

\section{Referencias}

Aboujaoude, E. (2010). Problematic Internet use: An overview. World Psychiatry, 9(2), 85-90. doi:10.1002/j.2051-5545.2010. tb00278.x

Adan, A. (2012). Impulsividad funcional y disfuncional en jóvenes con consumo intensivo de alcohol (binge drinking). Functional and dysfunctional impulsivity in young binge drinkers. Adicciones, 24(1), 17-22.

Aita S., Boettcher A., Slagel B., Holcombe J., Espenan M., King M. y Hill B. (2016). The relation between verbal fluency and executive functioning: an exploratory factor analysis (EFA) approach. Archives of Clinical Neuropsychology 31 (2016) 584-673. doi:10.1093/arclin/acw043

American Psychiatric Association (2013). Diagnostic and Statistical Manual of Mental Disorders (DSM-5). American Psychiatric Association.

Andreassen, C., Pallessen, S., Griffiths, M., Torsheim, T. y Sinha, R. (2018). The development and validation of the Bergen-Yale Sex Addiction Scale with a large national sample. Frontiers in Psychology, 9, 144. doi: 10.3389/fpsyg.2018.00144. 
Bailey, K., West, R. y Kuffel, J. (2013). What would my avatar do? Gaming, pathology and risky decision making. Frontiers in Psychology, 4, 1-10. doi: 10.3389/fpsyg.2013.00609

Balconi, M. y Finocchiaro, R. (2016). Deficit in rewarding mechanisms and prefrontal left/right cortical effect in vulnerability for internet addiction. Acta Neuropsychiatrica, 28(5), 272-285. doi: 10.1017/neu.2016.9

Balconi, M., Campanella, S. y Finocchiaro, R. (2017b). Web addiction in the brain: cortical oscillations, autonomic activity and behavioral measures. Journal of Behavioral Addiction, 6(3), 334-344. doi: $10.1556 / 2006.6 .2017 .041$

Bausela-Herreras, E. (2014). Funciones ejecutivas: nociones del desarrollo desde una perspectiva neuropsicológica. Acción Psicológica, 11(1), 21-34. doi: http://dx.doi.org/10.5944/ap.1.1.13789

Balconi, M., Venturella, I. y Finocchiaro, R. (2017a). Evidences from rewarding system, FRN and P300 effect in internet-addiction in young people. Brain Sciences, 7(81), 1-16. doi: 10.3390/brainsci7070081

Billieux J. y Van der Linden M. (2012). Problematic use of the Internet and self-regulation: a review of the initial studies. Open Addiction Journal, 5, 24-29. doi: 10.2174/1874941991205010024

Blakemore, S.J. y Choudhury, S. (2006). Development of the adolescent brain: implications for executive function and social cognition. Journal Child Psychological Psychiatry, 47(3-4), 296-312. doi: 10.1111/j.1469-7610.2006.01611.x.

Caplan, S. (2002). Problematic Internet use and psychosocial well-being: development of a theory-based cognitive-behavioral measurement instrument. Computer Human Behavior, 18, 553575.doi:10.1016/S0747-5632(02)00004-3.

Casey B., Giedd J. y Thomas K. (2000). Structural and functional brain development and its relation to cognitive development. Biological Psychology, 54, 241-257. doi:10.1016/S0301-0511(00)00058-2.

Casey, B. J., Tottenham, N., Liston, C., y Durston, S. (2005). Imaging the developing brain: what have we learned about cognitive development?. Trends in cognitive sciences, 9(3), 104-110. doi:10.1016/j.tics.2005.01.011

Chen Y. y Hsieh S. (2018) The relationship between internet-gaming experience and executive functions measured by virtual environment compared with conventional. Plos One, 13(6). doi.org/10.1371/journal.pone.0198339.

Chou, C., Condron, L. y Belland, J. (2005). A review of the research on Internet addiction. Educational Psychology Review, 17, 363-387. doi:10.1007/s10648-005-8138-1.

Cohen J. y Aston-Jones G. (2005). Cognitive neuroscience: decision amid uncertainty. Nature, 436, 471-2.

Davis, R. (2001). A cognitive-behavioral model of pathological Internet use. Computers in Human Behavior, 17, 187-195. doi:10.1016/S0747-5632(00)00041-8.

Dong, G. y Potenza, M. (2016). Risk-taking and risky decision-making in Internet gaming disorder: Implications regarding online gaming in the setting of negative consequences. Journal of Psychiatric Research, 73, 1-8. doi:10.1016/j.jpsychires.2015.11.011.

Dong, G., Li, H., Wang, L. y Potenza, M. (2017). Cognitive control and reward/loss processing in Internet gaming disorder: Results from a comparison with recreational Internet game-users. European Psychiatry, 44, 30-38. doi:10.1016/j.eurpsy.2017.03.004. 
Dong, G., Lin, X. y Potenza, M. (2015). Decreased functional connectivity in an executive control network is related to impaired executive function in Internet gaming disorder. Progress in NeuroPsychopharmacology and Biological Psychiatry, 57, 76-85. doi:10.1016/j.pnpbp.2014.10.012.

Dong, G., Lin, X., Zhou, H. y Lu, Q. (2014). Cognitive flexibility in internet addicts: fMRI evidence from difficult-to-easy and easy-to-difficult switching situations. Addictive Behaviors, 39(3), 677-683. doi: 10.1016/j.addbeh.2013.11.028.

Dong, G., Lu, Q., Zhou, H. y Zhao, X. (2010). Impulse inhibition in people with Internet addiction disorder: Electrophysiological evidence from a Go/NoGo study. Neuroscience Letters, 485(2), $138-142$. doi:10.1016/j.neulet.2010.09.002.

Dong, G., Zhou, H. y Zhao, X. (2011). Male Internet addicts show impaired executive control ability: Evidence from a color-word Stroop task. Neuroscience Letters, 499(2), 114-118. doi:10.1016/j.neulet.2011.05.047.

Dufour, M., Brunelle, N., Tremblay, J., Leclerc, D., Cousineau, M.-M., Khazaal, Y., Légaré, A., Rousseau, M. y Berbiche, D. (2016). Gender Difference in Internet Use and Internet Problems among Quebec High School Students. The Canadian Journal of Psychiatry, 61(10), 663-668. doi:10.1177/0706743716640755.

Ernst M. y Paulus M. (2005). Neurobiology of decision making: a selective review from a neurocognitive and clinical perspective. Biological psychiatry, 58, 597-604.

Fitzpatrick, S., Gilbert, S. J. y Serpell, L. E. (2013). Systematic review: are overweight and obese individuals impaired on behavioural tasks of executive functioning? Neuropsychol. Rev. 23, 138-156. doi: $10.1007 / \mathrm{s} 11065-013-9224-7$

García-Moreno, L.M., Expósito, J., Sanhueza, C. y Angulo, M. (2008). Prefrontal activity and weekend alcoholism in the young. Adicciones, 20 (3), 271-279. doi: 10.20882/adicciones.269.

Gogtay, N., Giedd, J., Lusk, L., Greenstein, D., Vaituzis, A.C., Nugent, T., Herman, DH. et al. (2004). Dynamic mapping of human cortical development during childhood through early adulthood. Proceedings of the National Academy Science of the United States of America, 101(21), 8174-8179.

Griffiths M.A. (2005). 'Components' model of addiction within a biopsychosocial framework. Journal of Substance Use, 10(4), 191-197.

Hasan, A., y Jaber, A. (2019). Prevalence of internet addiction, its association with psychological distress, coping strategies among undergraduate students. Nurse Education Today, 81, 78-82. doi:10.1016/j.nedt.2019.07.004.

Hansen, S. (2002). Excessive Internet usage or "Internet addiction"? The implications of diagnostic categories for student users. Journal of Computer Assisted Learning. 18, 235-236. doi:10.1046/j.13652729.2002.t01-2-00230.x.

He, W., Qi, A., Wang, Q., Wu, H., Zhang, Z., Gu, R., y Luo, W. (2017). Abnormal reward and punishment sensitivity associated with Internet addicts. Computers in Human Behavior, 75, 678-683. doi:10.1016/j.chb.2017.06.017.

Hekmat, S., Mehrjerdi, Z.A., Moradi, A., Ekhtiari, H. y Bakhshi, S. (2011) Cognitive Flexibility, Attention and Speed of Mental Processing in Opioid and Methamphetamine Addicts in Comparison with Non-Addicts. Basic \& Clinical Neuroscience 2(2): 12-19. 
Henry, J. D., y Crawford, J. R. (2004). Verbal fluency deficits in Parkinson's disease: a meta-analysis. Journal of International Neuropsychological Society, 10, 608-622. doi: 10.1017/S1355617704104141

Hirshorn E. y Thompson-Schill S. (2006). Role of the left inferior frontal gyrus in covert word retrieval: Neural correlates of switching during verbal fluency. Neuropsychologia, 44, 2547-2557.

Hsieh K., Hsiao R., Yang Y., Liu T. y Yen C. (2018). Predictive effects of sex, age, depression, and problematic behaviors on the incidence and remission of internet addiction in college students: a prospective study. International Journal of Environmental Research and Public Health, 15(12), 2861. doi: 10.3390/ijerph15122861

Ioannidis K, Chamberlain S., Treder M., Kiraly F., Leppink E., Redden S., Stein, D., Lochner, C. y Grant, J. Problematic internet use (PIU): associations with the impulsive-compulsive spectrum. An application of machine learning in psychiatry. Journal of Psychiatry Research, 83, 94-102.

Jeromin, F., Nyenhuis, N. y Barke, A. (2016). Attentional bias in excessive Internet gamers: Experimental investigations using an addiction Stroop and a visual probe. Journal of Behavioral Addictions, 5(1), 3240. doi:10.1556/2006.5.2016.012.

Jiang, Z., Zhao, X. y Li, C. (2017). Self-control predicts attentional bias assessed by online shopping-related Stroop in high online shopping addiction tendency college students. Comprehensive Psychiatry, 75, 1421. doi:10.1016/j.comppsych.2017.02.007

Jorgenson, A. , Hsiao, R. C. y Yen, C. (2016). Internet Addiction and Other Behavioral Addictions. Child and Adolescent Psychiatric Clinics of North America, 25(3), 509-520. doi:10.1016/j.chc.2016.03.004.

Király O, Griffiths MD, y Demetrovics Z. (2015) Internet gaming disorder and the DSM-5: conceptualization, debates, and controversies. Current Addiction Reports, 2, 254-62

Ko, C., Hsieh, T., Chen, C., Yen, C., Chen, C., Yen, J., Wang, P., y Liu, G. C. (2014). Altered brain activation during response inhibition and error processing in subjects with Internet gaming disorder: a functional magnetic imaging study. European Archives of Psychiatry and Clinical Neuroscience, 264(8), 661-672. doi:10.1007/s00406-013-0483-3.

Ko, C., Hsiao, S., Liu, G., Yen, J., Yang, M. y Yen, C. (2010). The characteristics of decision making, potential to take risks, and personality of college students with Internet addiction. Psychiatry Research, 175(1-2), 121-125. doi:10.1016/j.psychres.2008.10.004

Krishnamurthy S. y Chetlapalli S. (2015). Internet addiction: Prevalence and risk factors: A cross-sectional study among college students in Bengaluru, the Silicon Valley of India. Indian Journal of Public Health, 59 (2), 115-121.

Kuss, D., y Lopez-Fernandez, O. (2016). Internet addiction and problematic Internet use: A systematic review of clinical research. World Journal of Psychiatry, 6(1), 143-176. doi:10.5498/wjp.v6.i1.143.

Kuss, D., Griffiths, M., Karila, L., y Billieux, J. (2014). Internet addiction: A systematic review of epidemiological research for the last decade. Current pharmaceutical design, 20(25), 4026-4052.

Kuss, D., Griffiths, M. y Binder, J. (2013). Internet addiction in students: Prevalence and risk factors. Computers in Human Behavior, 29, 959-966.

Littel, M., van den Berg, I., Luijten, M., van Rooij, A. J., Keemink, L. y Franken, I. (2012). Error processing and response inhibition in excessive computer game players: an event-related potential study. Addiction Biology, 17(5), 934-947. doi:10.1111/j.1369-1600.2012.00467.x. 
Liu, T., y Potenza, M. N. (2007). Problematic Internet use: Clinical implications. CNS Spectrums, 12(6), 453466. doi:10.1017/S1092852900015339

Luengo-López, A. (2004). Adicción a Internet: conceptualización y propuesta de intervención. Revista profesional española de terapia cognitivo-conductual, 2(1), 22-52.

Marazziti, D., Catena Dell'Osso, M., Conversano, C., Consoli, G., Vivarelli, L., Mungai, F., Di Nasso, E. y Golia, F. (2008). Executive function abnormalities in pathological gamblers. Clinical Practice and Epidemiology in Mental Health, 4(1), 7. doi:10.1186/1745-0179-4-7.

Martínez-Selva, J., Sánchez-Navarro, J., Bechara, A. y Román, F. (2006). Mecanismos cerebrales de la toma de decisiones. Revista de Neurología 42, 411-8. https://www.neurologia.com/articulo/2006161.

Meerkerk, G., Van Den Eijnden, R., Franken, I. y Garretsen, H. (2010). Is compulsive Internet use related to sensitivity to reward and punishment and impulsivity? Computers in Human Behavior, 26, 729-735. doi:10.1016/j.chb.2010.01.009.

Meerkerk, G.-J., Van Den Eijnden, R. J. J. M., Vermulst, A. A., y Garretsen, H. F. L. (2009). The compulsive internet use scale (CIUS): Some psychometric properties. CyberPsychology and Behavior, 12(1), 1-6

Meerkerk, G.,Van Den Eijnden, R. y Garretsen, H. (2006). Predicting compulsive Internet use: it's all about sex! Cyberpsychology Behavior, 9, 95-103. doi:10.1089/cpb.2006.9.95.

Meerkerk, G., Van Den Eijnden, R., Vermulst,A. y Garretsen, H. (2009). The Compulsive Internet Use Scale (CIUS): some psychometric properties. Cyberpsychology Behavior, 12, 1-6. doi:10.1089/cpb.2008.0181.

Nie, J., Zhang, W. y Liu, Y. (2016). Exploring depression, self-esteem and verbal fluency with different degrees of internet addiction among Chinese college students. Comprehensive Psychiatry, 72, 114-120. doi:10.1016/j.comppsych.2016.10.006

Park, S., Hong, K. M., Park, E., Ha, K., y Yoo, H. (2013). The association between problematic Internet use and depression, suicidal ideation and bipolar disorder symptoms in Korean adolescents. Australian \& New Zealand Journal of Psychiatry, 47(2), 153-159. doi:10.1177/ 0004867412463613.

Pawlikowski, M. y Brand, M. (2011). Excessive Internet gaming and decision making: Do excessive World of Warcraft players have problems in decision making under risky conditions? Psychiatry Research, 188(3), 428-433. doi:10.1016/j.psychres.2011.05.017.

Ríos-Lago M., Periáñez J.A. y Muñoz-Céspedes J.M (2004). Attentional control and slowness of information processing after severe traumatic brain injury. Brain Inj, 18: 257-272.

Rothbart, M. K., y Posner, M. I. (2015). The developing brain in a multitasking world. Dev Rev, 35, 42-63. doi: 10.1016/j.dr.2014.12.006

Saville, B. K., Gisbert, A., Kopp, J. y Telesco, C. (2010). Internet Addiction and Delay Discounting in College Students. The Psychological Record, 60(2), 273-286. doi:10.1007/bf03395707.

Seok, J., y Sohn, J. (2018). Altered prefrontal and inferior parietal activity during a stroop task in individuals with problematic hypersexual behavior. Frontiers in Psychiatry, 9. doi:10.3389/fpsyt.2018.00460.

Shao, Y., Zheng, T., Wang, Y., Liu, L., Chen, Y. y Yao, Y. (2018). Internet addiction detection rate among college students in the People's Republic of China: a meta-analysis. Child and Adolescent Psychiatry and Mental Health, 12(1). doi:10.1186/s13034-018-0231-6.

Shaw, M. y Black, D.W. (2015). Internet addiction. CNS Drugs, 22(5), 353-365.

Spada, M. (2014). An overview of problematic Internet use. Addictive Behavior, 39 (1), 3-6. 
Steward, T., Mestre-Bach, G., Vintró-Alcaraz, C., Lozano-Madrid, M., Agüera, Z., Fernández-Formoso, J. A., et al. (2018). Food addiction and impaired executive functions in women with obesity. European Eating Disorders Review, 26(6), 574-584. doi: 10.1002/erv.2636.

Su, W., Potenza, M. N., Zhang, Z., Hu, X., Gao, L. y Wang, Y. (2018). Do individuals with problematic and non-problematic internet game use differ in cooperative behaviors with partners of different social distances? Evidence from the Prisoner's Dilemma and Chicken Game. Computers in Human Behavior, 87, 363-370. doi: 10.1016/j.chb.2018.05.040.

Sun, D., Chen, Z., Ma, N., Zhang, X., Fu, X. y Zhang, D. (2009). Decision-Making and Prepotent Response Inhibition Functions in Excessive Internet Users. CNS Spectrums, 14(02), 75-81. doi: $10.1017 / \mathrm{s} 1092852900000225$.

Tao, R., Wang, J., Huang, X., Liu, C. Y., Yao, S., Xiao, L., et al. (2008). The naming, definition and clinical diagnostic criteria of internet addiction. Medical Journal of the Chinese People's Armed Police Forces, 19(9), 773-776.

Tekin, A., Yetkin, A., Adigüzel, S. y Akman, H. (2018). Evaluation of Stroop and Trail-Making Tests performance in university students with internet addiction. Anatolian Journal of Psychiatry, 19(6), 593598. doi:10.5455/apd.292389.

Tranel D. y Damasio A.R. Neuropsychology and behavioral neurology. In Cacioppo J.T., Tassinary L.G., Berntson G.G,. eds. Handbook of psychophysiology, 2 ed. New York: Cambridge University Press; 2000. p. 119-141.

Wang, L., Zhang, Y., Lin, X., Zhou, H., Du, X. y Dong, G. (2017d). Group independent component analysis reveals alternation of right executive control network in Internet gaming disorder. CNS Spectrums, 23(5), 300-310. doi:10.1017/s1092852917000360.

Wang, Y., Hu, Y., Xu, J., Zhou, H., Lin, X., Du, X. y Dong, G. (2017c). Dysfunctional Prefrontal Function Is Associated with Impulsivity in People with Internet Gaming Disorder during a Delay Discounting Task. Frontiers in Psychiatry, 8, 1-11. doi:10.3389/fpsyt.2017.00287.

Wang, Y., Wu, L., Wang, L., Zhang, Y., Du, X. y Dong, G. (2017b). Impaired decision-making and impulse control in Internet gaming addicts: evidence from the comparison with recreational Internet game users. Addiction Biology, 22(6), 1610-1621. doi:10.1111/adb.12458.

Wang, Y., Wu, L., Zhou, H., Lin, X., Zhang, Y., Du, X. y Dong, G. (2017a). Impaired executive control and reward circuit in Internet gaming addicts under a delay discounting task: independent component analysis. European Archives of Psychiatry and Clinical Neuroscience, 267(3), 245-255. doi:10.1007/s00406-016-0721-6.

White, H., Marmorstein, N., Crews, F., Bates, M., Mun, E. y Loeber, R. (2011). Associations between heavy drinking and changes in impulsive behavior among adolescent boys. Alcoholism: Clinical and Experimental Research, 35(2), 295-303.

Whiteside D., Kealey, T, Semla, M., Luu, H., Rice, L., Basso, M., Roper y B. (2016) Verbal Fluency: Language or Executive Function Measure? Applied Neuropsychology Adult, 23(1), 29-34. doi: 10.1080/23279095.2015.1004574. 
Wu, A. M. S., Cheung, V. I., Ku, L. y Hung, E. P. W. (2013). Psychological risk factors of addiction to social networking sites among Chinese smartphone users. Journal of Behavioral Addictions, 2(3), 160-166. doi:10.1556/jba.2.2013.006.

Widyanto, L. y Griffiths, M.D. (2006). Internet addiction: a critical review. International Journal of Mental Health and Addiction, 4, 31-51. doi:10.1007/s11469-006-9009-9.

Xing, L., Yuan, K., Bi, Y., Yin, J., Cai, C., Feng, D., et al. (2014). Reduced fiber integrity and cognitive control in adolescents with internet gaming disorder. Brain Research, 1586, 109-117. doi:10.1016/j.brainres.2014.08.044.

Xue G., Lu Z., Levin I.P. y Bechara A. (2011) An fMRI study of risk-taking following wins and losses: implications for the gambler's fallacy. Human brain mapping, 32, 271-281.

Yao, Y. W., Chen, P. R., Chen, C., Wang, L. J., Zhang, J. T., Xue, G., Deng, L., Liu, Q., Yip, S. y Fang, X. (2014). Failure to utilize feedback causes decision-making deficits among excessive Internet gamers. Psychiatry Research, 219(3), 583-588. doi:10.1016/j.psychres.2014.06.033.

Yao, Y. W., Chen, P. R., Li, S., Wang, L. J., Zhang, J. T., Yip, S. W., Chen G., Deng, L., Liu, Q. y Fang, X. (2015). Decision-Making for Risky Gains and Losses among College Students with Internet Gaming Disorder. PloS one, 10(1), e0116471. doi:10.1371/journal.pone.0116471.

Young, K. (1998). Internet addiction: the emergence of a new clinical disorder. Cyberpsychology \& Behavior, 1(3), 237-244. doi: 10.1089/cpb.1998.1.237.

Young, K. (2004). Internet addiction: a new clinical phenomenon and its consequences. American Behavioral Scientist, 48(4), 402-415. doi: 10.1177/0002764204270278.

Young, K.S., Yue, X.D. y Ying, L. (2011). Prevalence estimates and etiologic models of Internet addiction. En Internet Addiction (pp.3-18). New Jersey: John Wiley \& Sons.

Zhang, Y., Lin, X., Zhou, H., Xu, J., Du, X. y Dong, G. (2016). Brain Activity toward Gaming-Related Cues in Internet Gaming Disorder during an Addiction Stroop Task. Frontiers in Psychology, 7, 1-9. doi:10.3389/fpsyg.2016.00714.

Recibido: 30-03-2020

Aceptado: 17-07-2020 